\title{
Aktuelle Forschungsfragen der deutschsprachigen Phraseodidaktik
}

\author{
Erla Hallsteinsdóttir (Odense)
}

\begin{abstract}
Phraseology in foreign language learning and teaching has been an object of linguistic and didactic research for almost 40 years. This article begins with an overview over phraseodidactic research in German. Then it discusses aspects of phraseology in teaching German as a foreign language, i.a. the influence of Kühn's three step model on phraseology in foreign language teaching and methods to define the phraseological units that are to be learned (phraseological minimum). Finally, it addresses a newer research approach: The question how multimodality affects the language competence and thereby also the learning of phraseology.
\end{abstract}

\section{$1 \quad$ Einleitung}

Die Phraseologieforschung hat sich in den letzten 40 Jahren $\mathrm{zu}$ einer eigenständigen, allgemein anerkannten Forschungsdisziplin entwickelt, in der interdisziplinäre Forschungsansätze zu vielfältigen Fragestellungen aus verschiedenen theoretischmethodischen Paradigmen heraus verfolgt werden. Neben allen Bereichen der theoretischen Sprachwissenschaft und den angewandten Disziplinen Translatologie, Lexikografie und Sprachdidaktik spielen Forschungsansätze in Disziplinen wie Literaturwissenschaft, Kulturwissenschaft, Kommunikationswissenschaft, Ethnologie, Kunstgeschichte, Psychologie, Jura, Mathematik, Informationstechnologie u.a. eine immer größere Rolle in der Phraseologie ${ }^{1}$.

Die phraseologische Grundforschung liefert u.a. Material für angewandte Arbeiten, z.B. Wörterbücher und Unterrichtsmaterial und Erkenntnisse zu Voraussetzungen sowohl von Fremdsprachenlernern als auch zu sprachsystematischen Eigenschaften der Muttersprache und der Fremdsprache sowie zu textsorten-, sprecher-, und sprachspezifischen Verwendung von Phraseologie. Solche Forschungsergebnisse bilden die Grundlage für die Phraseodidaktik, d.h. die systematische Vermittlung von Phraseologie (cf. Ettinger 2001: 87). Nicht zuletzt der verstärkte Fokus der Phraseologieforschung auf kognitive Fragestellungen dürfte in den kommenden Jahren eine fruchtbare Grundlage der Phraseodidaktik bilden.

\footnotetext{
1 Einen guten Überblick über den Objektbereich der Phraseologie gibt Burger (2007). Die Publikationsübersicht der Internetseite von EUROPHRAS (http://www.europhras.org/ gesehen am 26. September 2010), die Idiom-Bibliografie des Projekts Kollokationen im Wörterbuch (http://kollokationen.bbaw.de/bib/a.html gesehen am 26. September 2010) und die Konferenzbände zu den EUROPHRASKonferenzen zeigen die Vielfältigkeit aktueller Forschungsaktivitäten und -ansätze innerhalb der Phraseologieforschung. Die Abstracts von EUROPHRAS 2004 in Basel findet man hier: http://www.sprachwissenschaft.ch/europhras/Abstracts.pdf und von EUROPHRAS 2008 in Helsinki hier: http://www.helsinki.fi/saksa/europhras/einladung.htm und ein Teil der Beiträge von EUROPHRAS 2008 ist online veröffentlicht worden: http://www.helsinki.fi/deutsch/europhras/ep2008.pdf. Das Programm von EUROPHRAS 2010 in Granada findet man hier: http://www.ugr.es/ apamies/horario-comunicaciones.pdf (alle gesehen am 26. September 2010).
} 
In diesem Beitrag wird zuerst ein kurzer Überblick über einige Forschungsbereiche der deutschsprachigen Phraseodidaktik gegeben und anschließend etwas ausführlicher auf folgende Aspekte eingegangen:

1. Ansätze des Lehrens und Lernens von Phraseologie;

2. den phraseologischen Dreischritt und seinen Einfluss in der Phraseodidaktik;

3. die Erarbeitung eines phraseologischen Grundwortschatzes für Deutsch als Fremdsprache und

4. die phraseologische Kompetenz in Relation zum Phänomen Multimodalität, d.h. das Zusammenspiel zwischen sprachlichen und nicht-sprachlichen Aspekten in der Phraseologie in Hinblick auf ihre Relevanz im Fremdsprachenunterricht.

Abschließend und als Übergang zu den weiteren Beiträgen dieses Themenheftes wird zusammenfassend ein Fragekatalog mit aktuellen Forschungsfragen der Phraseodidaktik formuliert.

\section{Forschungsbereiche der Phraseodidaktik}

\subsection{Ein kurzer Überblick über die deutschsprachige Phraseodidaktik}

Die Phraseodidaktik ist

[...] der Teilbereich der Phraseologie, der sich mit der systematischen Vermittlung von

Phraseologismen im mutter- oder fremdsprachlichen Unterricht befasst. (Ettinger 2001: 87)

Im Folgenden bildet die deutschsprachige Phraseodidaktik, die eine inzwischen gut 20 jährige Tradition ${ }^{2}$ vorzuweisen hat ${ }^{3}$, den Gegenstand meiner Betrachtungen (cf. ausführlich zu ihren inhaltlichen Schwerpunkten in Ettinger 2007). Größere Publikationen, die sich dem Thema Phraseodidaktik widmen, sind noch immer relativ selten. Neben dem Themenheft von Wotjak (1996) und den Sammelbänden von Lorenz-Bourjot/Lüger (2001) bzw. Jesenšek/Fabčič (2007) gibt es eine Anzahl Konferenz- und Zeitschriftenbeiträge (cf. Lüger 1997 und die Bibliographie in Lüger1997a ${ }^{4}$ ). Im internationalen Handbuch zur Phraseologie geben Kühn (2007) und Häcki/Buhofer (2007) einen Überblick über Phraseologie im Muttersprachenunterricht respektive Erstspracherwerb, während Ettinger (2007) und Wray (2007) die Situation der Phraseologie im Fremdsprachenunterricht darstellen.

Zur Wichtigkeit der Phraseologie im Fremdsprachenunterricht werden verschiedene Meinungen vertreten. Lange wurde davon ausgegangen, dass Phraseologismen Randerscheinungen sind, die in der fremdsprachigen Kommunikation nicht unmittelbar notwendig sind. Einzelne Forscher vertreten immer noch die Meinung, dass bestimmte Typen von Phraseologismen, wenn überhaupt, dann erst ,[a]b einer weit fortgeschrittenen oder gar „near-native“-Lernstufe“ (Schmale 2009: 175-176) relevant sind. In den letzten Jahren hat jedoch der Standpunkt immer mehr Anhänger gewonnen, dass Phraseologismen für die fremdsprachliche Kommunikation unverzichtbar sind, dass sogar eine begrenzte Kommunikation in der Fremdsprache ohne eine minimale Beherrschung der Phraseologie nicht möglich ist (cf. Daniels/Pommerin 1979: 575; W. Fleischer 1997: 32; Gülich/Krafft 1992: 82; Kühnert 1985: 223). Dies gilt insbesondere für Phraseologie im weiten Sinne, zu der grundlegende Wortschatzeinheiten wie Kollokationen und Routineformeln gezählt

\footnotetext{
2 Als einen bahnbrechenden Vorreiter der Phraseodidaktik nennt Ettinger (1998: 205) die Arbeit von D. Götz (1976): Stilistik und Idiomatik im Englischunterricht.

${ }^{3}$ In der englischsprachigen Forschung ist das Gebiet jedoch deutlich jünger und setzt z.T. andere Schwerpunkte.

${ }^{4}$ http://www.ub.uni-konstanz.de/kops/volltexte/1999/295/pdf/295_1.pdf (gesehen am 28. September 2010).
} 
werden. Dem Mythos der Nicht-Akzeptabilität der Verwendung von Phraseologie ${ }^{5}$ steht die zwingende Notwendigkeit des Erwerbs von fremdsprachlicher Phraseologie gegenüber:

Hinsichtlich der Frage nach der Behandlung formelhafter Sprache im Fremdsprachenunterricht ist daher festzuhalten, dass ihre Vermittlung eine absolute Notwendigkeit darstellt. Da vorgefertigte Formulierungen für die soziale Interaktion eine zentrale Rolle spielen, ist ihre angemessene Verwendung unentbehrlich für die Akzeptanz durch die Sprecher der Zielsprachengemeinschaft. Aus diesem Grund kommt dem Erwerb und dem Gebrauch formelhafter Sprache durch Nicht-Muttersprachler eine wichtige Bedeutung zu. (Aguado 2002:

Dieser Ausgangspunkt führt dazu, dass die Kenntnisse der fremdsprachlichen Phraseologie als ein wichtiger Maßstab der fremdsprachlichen Kompetenz, als ,ein Qualitätsmerkmal der Sprachbeherrschung“ (Ulbricht 1989: 98) und das Lernen von Phraseologismen als eine grundlegende Voraussetzung der Sprachbeherrschung angesehen werden. Je mehr Phraseologismen ein Fremdsprachler kennt, desto besser ist seine fremdsprachliche Sprachkompetenz (cf. Hessky 1997: 139; cf. auch Glaap 1985: 96).

In den Anfängen der Phraseodidaktik wurden Phraseologismen im Fremdsprachenerwerb und im Fremdsprachenunterricht meist im Zusammenhang mit Lernschwierigkeiten und Fehlern untersucht und in der Fremdsprachendidaktik wurden Phraseologismen ihrer phraseologiespezifischen Besonderheiten wegen ebenfalls häufig als ein spezielles Problem behandelt (cf. Bergerová o.J.; Eismann 1979: 565; Günthner 1991: 415; Lüger 1997; Stedje 1987; Ulbricht 1989: 99 ff.). Die Phraseologie gilt nach wie vor als ,kein leicht durchschaubares Phänomen“ (Wotjak 1992: 104), das „für den Fremdsprachenlerner schwer zu überwindende Verständnis-, Lern- und Anwendungsprobleme“ (Weller 1992: 129) darstellt. Es wird vielfach angenommen, dass fremdsprachliche Phraseologismen als vorgeformte semantische und syntaktische Ganzheiten auswendig gelernt werden müssen, um sie verstehen und anwenden zu können (cf. Koller 1987: 109; Kühnert 1985: 225; Roos 1992: 194 ff.; Weller 1992: 134). Die Resultate der Untersuchung von Hallsteinsdóttir zeigen jedoch,

... dass das Verstehen sowohl unbekannter als auch geläufiger idiomatischer fremdsprachlicher Phraseologismen im Diskurs prinzipiell möglich ist, vorausgesetzt, der Sprecher verfügt über ein Minimum an Sprachwissen in der Fremdsprache. [...] Wenn die Bedeutung der einzelnen Komponenten bekannt ist oder die phraseologische Bedeutung durch Informationen aus dem Kontext indiziert wird, kann in der Regel eine sinnkonstante phraseologische Bedeutung konstruiert werden. (Hallsteinsdóttir 2001: 308)

Das eine schließt jedoch das andre nicht unbedingt aus, denn wie Aguado (2002: 40) hervorhebt, so können ganzheitlich gelernte Phraseologismen eine zentrale Funktion beim Fremdsprachenlernen haben

\footnotetext{
5 In Schmale (2009: 151-152) wird eine Auffassung von „Kulturemen“ übernommen, die eine Nicht-Akzeptanz der Verwendung von Phraseologismen seitens der Muttersprachler bezeichnet:

\begin{abstract}
Von Nicht-Muttersprachlern wird nämlich Dobrovol'skij/Lûbimova (1993) zufolge eine zu starke Assimilation an die zielsprachliche Kulturgemeinschaft, die durch eine Verwendung besonders bildhaft-metaphorischer Idiome gegeben sein könnte, nicht erwartet bzw. sogar kommunikativ sanktioniert. Natürlich vor allem dann, wenn Fremdsprachensprecher diese Assimilation auf phonetischer, syntaktischer, semantischer oder auch pragmatischer Ebene nicht in gleichem Maße vollzogen haben, d.h. sich durch einen Muttersprachlern nicht bekannten Akzent oder bestimmte strukturelle oder pragmatische „Fehler“ als Nicht-Muttersprachler zu erkennen geben. Doch selbst wenn Letzteres nicht mehr der Fall ist, bestehen berechtigte Zweifel daran, dass bestimmte Verwendungsweisen von Idiomen, insbesondere Wortspiele mit Phrasemen, von Nicht-Muttersprachlern kommentarlos akzeptiert werden.
\end{abstract} (Schmale 2009: 151-152)
}

M.E. handelt es sich hierbei um einen Mythos, der immer wieder ohne Hinterfragung der dieser Behauptung zugrunde liegenden Daten und ohne eine empirische Überprüfung überliefert wird. 
insofern, als sie - je nach Art der Sequenz - sowohl die grammatische als auch die pragmatische bzw. idiomatische Kompetenz verbessern helfen. Die Segmentierung dieser Sequenzen zwecks Extraktion grammatischer Information durch den Lernenden ist für den langfristigen Erwerb zentral und ein wesentliches Merkmal produktiver Kompetenz. Wenn die L2-Lernenden erkennen, dass die von ihnen gebrauchten komplexen Sequenzen aus kleineren Einheiten zusammengesetzt sind, sie diese entsprechend segmentieren, die zwischen den auf diese Weise gewonnenen Einheiten bestehenden Beziehungen erkennen und analysieren und die Einheiten schließlich produktiv in anderen Kontexten und Konstruktionen verwenden, findet ein direkter Einfluss formelhafter Sequenzen auf den Erwerb grammatischen Wissens statt. Die genannten Schritte (Segmentierung und Analyse) sind somit die Voraussetzung für die Re-Strukturierung des lernersprachlichen Systems. (Aguado 2001: 40)

Sowohl beim Verstehen als auch in der Sprachproduktion werden unterschiedliche Strategien eingesetzt, die möglicherweise als regelbasierte Verfahren erfasst und für den Einsatz im Fremdsprachenunterricht beschrieben werden können (cf. Hallsteinsdóttir 2001). Aguado nimmt an,

dass auch Formelhaftigkeit und Kreativität koexistent sind bzw. in einem arbeitsteiligen Verhältnis zueinander stehen und auch miteinander interagieren. (Aguado 2002: 42)

Um eine kommunikativ angemessene Sprachkompetenz aufzubauen wäre daher der Phraseologie im weiten Sinne sowie den mit der Verarbeitung von Phraseologismen verbundenen Strategien eine zentrale Rolle im Fremdsprachenunterricht einzuräumen (cf. ausführlich zu Forschungsdesiderata in Aguado 2002; $\mathrm{zu}$ Verstehensstrategien in Hallsteinsdóttir 2001; zur Vermittlung metasprachlicher Strategien in Bergerová 2009, 2010; Jesenšek 2006, sowie zur phraseologischen Kompetenz im Abschnitt 2.5 weiter unten).

\subsection{Ansätze des Lehrens und Lernens von Phraseologie}

Inzwischen gibt es mehrere Ansätze, das Lernen von Phraseologismen in speziellen Übungen oder Unterrichtsmaterialien systematisch didaktisch aufzuarbeiten. Hier sind z.B. die Darstellungen in B. Wotjak (1996), die Überlegungen zur Phraseodidaktik bei Ettinger (1998 2001) sowie die Überlegungen zur Phraseologie in Lehrwerken Deutsch als Fremdsprache und zu speziellen Übungsbüchern vgl. Kühn (1992, 170 ff.) zu nennen. Das vor fast 20 Jahren gefällte Urteil von Kühn ist vernichtend:

Übungen zu Phraseologismen sind sowohl in den Lehrwerken DaF als auch in den speziellen Arbeitsbüchern nur Kontrolltests, anhand derer der Lehrer feststellen kann, ob der Schüler die Struktur und Bedeutung der Phraseologismen kennt oder nicht. Struktur und Bedeutung von Phraseologismen über solche Kontrollübungen zu lernen, ist Vokabeldrill in Quizform und wird den sprachlichen Besonderheiten von Phraseologismen nicht gerecht. (Kühn 1992: 177)

Zehn Jahre später stellen Lorenz-Bourjot/Lüger (2001: V) im Vorwort zu ihrem Buch Phraseologie und Phraseodidaktik fest, dass es eine Tendenz zur „Betonung (re-)integrativer Ansätze", darunter auch in Richtung angewandte Disziplinen, gibt. Diese Tendenz ist schon im Themenheft von Wotjak (1996) zu sehen und wird mit dem Band von LorenzBourjot/Lüger weitergeführt. Dieser liefert mit dem zweiten Themenbereich „Phraseodidaktik - Vom Wortschatzlernen zur Textarbeit“ (Lorenz-Bourjot/Lüger 2001: VI) sechs Beiträge zur Phraseodidaktik (Babillon 2001; Ettinger 2001; Henk 2001; Köster 2001; Lenk 2001; Stolze 2001), die „sich weitgehend auf Überlegungen zum Erlernen von Phraseologismen“ in ihren verwendungsspezifischen Kontexten konzentrieren.

Neben solchen integrativen Ansätzen zum Erlernen von Phraseologie mithilfe von Texten sind im Zeitalter der neuen Medien v.a. die neueren phraseodidaktischen Arbeiten und Lehrmaterialien hervorzuheben, die u.a. in diesem Themenheft in den Beiträgen von Bergerová, Jesenšek, Rentel, Krajl und Kacjan vorgestellt werden. Diese zeichnen sich durch die Einbeziehung komplexer Übungs- und Beschreibungsverfahren aus, die z.T. kontrastiv 
angelegt sind und die muttersprachlichen Voraussetzungen der jeweiligen DaF-Lerner berücksichtigen.

Als das wichtigste Forschungsdesiderat der fremdsprachlichen Phraseodidaktik nennt Ettinger

die Erarbeitung von Lehrbüchern [...], mit denen der aktive Gebrauch von Phrasemen im

Fremdsprachenunterricht vermittelt wird. (Ettinger 2007: 904)

Neben der Erarbeitung von solchen phraseologischen Lehrwerken besteht noch eine wichtige Aufgabe der Phraseodidaktik darin, eine Integration der Phraseologie in die allgemeinen Lehrwerke für Deutsch als Fremdsprache voranzutreiben. Das Postulat, dass Phraseologismen in die Lehrwerke und in den Unterricht zu integrieren sind, basiert darauf,

dass Phraseme übliche Elemente des Wortschatzes sind, die im Sprachunterricht parallel mit der Einwortlexik zu behandeln sind. (Jesenšek 2006: 144)

Denn Farøs Untersuchungen zu Funktionen der Phraseologie in literarischen Texten haben keinen grundlegenden funktionalen Unterschied zu Wörtern gezeigt (cf. Farø 2006), d.h. Phraseologismen werden bis auf wenige Ausnahmen als ,normale“ Sprachzeichen verwendet. Hier wird von der Hypothese ausgegangen, dass dies mit wenigen Ausnahmen (cf. die Textsorte Talkshow in Schmale 2009) für die meisten Textsorten gilt. Die Ausnahmen sind zugegeben die Verwendungsweisen, die den Sprechern auffallen. Bei solchen Verwendungen gibt es fast immer eine Einbeziehung der „Phraseoaktivität“" oder des (semantischen) Mehrwerts (cf. Koller 2007) in einem konkreten, dafür geschaffenen Kontext und mit bestimmten Funktionen (cf. z.B. Ptasnyk 2009, Sandig (2010) und Vajičková (2010) zu Funktionen von Modifikationen in deutschsprachigen Pressetexten).

Ein nach wie vor bestehendes Desiderat ist der phraseodidaktische Einsatz von Wörterbüchern im DaF-Unterricht (cf. Hallsteinsdóttir 2006: 116 ff.). Hier fehlen sowohl Forschungsarbeiten in der Phraseologie und Lexikografie als auch Konzeptionen für die didaktische Umsetzung (cf. jedoch Bergerová in diesem Heft).

\subsection{Der phraseologische Dreischritt}

Sehr viele phraseodidaktische Ansätze gehen auf die wegbereitenden Publikationen von Kühn (1992, 1994) zurück. Basierend auf theoretischen Auffassungen und praktischen Unterrichtserfahrungen hat Kühn den phraseologischen Dreischritt, ein Lernverfahren für den Erwerb fremdsprachlicher Phraseologismen für den Fremdsprachenunterricht, entwickelt (cf. im Folgenden Kühn 1992: 178 ff., 1994: 424 ff.; auch Ulbricht 1989: 102). Der phraseologische Dreischritt beinhaltet:

1. das Erkennen einer Wortverbindung im Text als phraseologische Einheit soweit dies möglich ist (cf. dazu auch Hallsteinsdóttir 1999; Lipold 1998);

2. das Entschlüsseln der phraseologischen Bedeutung durch das Bild, durch den Kontext oder durch das Nachschlagen in Wörterbüchern;

3. das Erwerben des Phraseologismus als eine vorgeformte Einheit von Form und Bedeutung für die Anwendung in bestimmten Kommunikationssituationen.

Die auf dem phraseologischen Dreischritt aufbauenden Lernschritte Erkennen, Verstehen, Festigen und Anwenden gelten heute in der Phraseodidaktik als die grundlegenden Phasen des Erwerbs von Phraseologismen (cf. ausführlich dazu in Ettinger 2007):

Bei der Behandlung von Phrasemen im Fremdsprachenunterricht stehen das Erkennen, Erlernen, Einüben, Behalten und die situationsadäquate Anwendung von Phrasemen im Vordergrund. (Ettinger 2007: 893) 
Verschiedene weitere Schritte sind auch inzwischen hinzugefügt worden, wie z.B. „eine Festigungsphase durch kontextualisierte Übungen“" (Bergerová 2010: 393-394) oder die Erweiterung von Laskowski, in der der Ansatz von Kühn in

vier Phasen: Bekanntmachen (Input), Erkennen, Enkodieren (Verarbeitung) und Gebrauch (Output- Reproduktion) von Ph. (Laskowski 2010: 403)

aufgeteilt wird.

Das didaktische Konzept des phraseologischen Dreischritts spielt bei der Entwicklung von neuerem Unterrichtsmaterial wie den Übungen der Sprichwort-Plattform ${ }^{6}$ (cf. Jesenšek in diesem Heft), Hana Bergerovàs Lehr- und Übungsmaterial ${ }^{7}$ (cf. Bergerovà in diesem Heft) und der Datenbank und Übungen von Stefan Ettinger ${ }^{8}$ sowie der EPHRAS-Datenbank ${ }^{9}$ (cf. Kralj/Kacjan in diesem Heft) eine große Rolle.

\subsection{Phraseologischer Grundwortschatz Deutsch als Fremdsprache}

Köster (2001: 149) bescheinigt der Phraseodidaktik eine Art Dämmerzustand, der an den in den 80er Jahren von Kühn diagnostizierten ,phraseologischen Dornröschenschlaf“ der Fremdsprachendidaktik Deutsch denken lässt:

die Behandlung von Phraseologismen im gesteuerten Spracherwerbskontext steht heute zwar außer Diskussion, es gibt aber keinen breiten Konsens über Auswahl (Phraseologieklassen, Phraseologismen im produktiven oder rezeptiven Wortschatz, phraseologisches Minimum oder Optimum) oder Methodik. (Köster 2001: 149) ${ }^{10}$

Die Bestimmung eines phraseologischen Grundwortschatzes für DaF, auf den sich Köster bezieht, wird seit 30 Jahren für die deutsche Sprache gefordert und z.T. auch umgesetzt (cf. Jesenšek in diesem Heft sowie Überlegungen zur Vermittlung eines phraseologischen/parömiologischen Minimums bei Baur et al. 1996; Daniels 1985; Durčo 2001; Eismann 1979; Häcki Buhofer/Burger 1994; Hallsteinsdóttir/Šajanková/Quasthoff 2006; Hessky 1992; Weller 1979). Die Kriterien und Arbeitsverfahren sind klar:

1. Aktualität: Die Auswahl soll auf der Basis von Frequenz (Sprachverwendung) und Geläufigkeit (Sprecherperspektive) erfolgen (cf. Jesenšek 2006: 142). Die Frequenz wird durch Korpusanalysen und die Geläufigkeit durch Informantenbefragungen bestimmt. Diese Verfahren können durch die statistische Kookkurrenzanalyse (cf. Steyer 2003) sowie die Intuition des Linguisten ergänzt werden (cf. eine ausführliche Darstellung und weitere Literaturverweise dazu in Hallsteinsdóttir/Šajanková/Quasthoff 2006: 118).

2. Relevanz: Die Auswahl der Phraseologismen soll sich an den im gemeinsamen europäischen Referenzrahmens für Sprachen (GER) vorgegebenen Themen orientieren. Bei der didaktischen Aufarbeitung sollen die Phraseologismen nach ihrer Zugehörigkeit zu Fertigkeiten (Sprechen, Hören, Lesen, Schreiben, Interaktion) und zu erlernenden Sprachhandlungen in die Niveaustufen des GER eingeordnet werden.

Neben diesen Kriterien spielt - im Idealfall - die Muttersprache der Lernenden eine Rolle für die Auswahl und Aufarbeitung der Phraseologismen. So fordert Hessky die Erarbeitung eines phraseologischen Optimums, in dem die Phraseologismen "in Abhängigkeit von der

\footnotetext{
$6 \mathrm{http}: / /$ www.sprichwort-plattform.org/sp/Sprichwort-Plattform (gesehen am 26. September 2010)

$7 \mathrm{http} / / /$ frazeologie.ujepurkyne.com/VS_teil04_ger.asp (gesehen am Juli 2010)

$8 \mathrm{http} / / / \mathrm{www}$.ettinger-phraseologie.de/pages/deutsche-redewendungen/uebungen.php (gesehen am Juli 2010)

${ }^{9}$ www.ephras.org (gesehen am 26. September 2010).

${ }^{10}$ Daraufhin fordert er eine generelle „Wortschatzwende in der DaF-Didaktik“ (Köster 2001: 49), die er mit einem Zitat von Michael Lewis begründet, dass Sprache aus grammatikalisierter Lexik bestehe, und nicht aus lexikalisierter Grammatik.
} 
Muttersprache der Lernenden, auf kontrastiver Basis und unter Zielsetzung, Lernstufe etc." (Hessky 1992: 167) aufbereitet werden. Dass sich die Einbeziehung der Muttersprache in DaF-Lehrwerken für Lerner mit unterschiedlichen Muttersprachen als schwierig bis unmöglich gestaltet, steht außer Frage. In Lehr- und Lernmaterialien, die für eine homogene Lernergruppe innerhalb einer Sprachgemeinschaft geschrieben werden, ist ein vergleichender Blick auf die Phraseologie jedoch durchaus möglich und sinnvoll, um Interferenzen aus der Muttersprache zu verhindern (cf. Hallsteinsdóttir 2001; Reder 2006, 2008, in diesem Heft).

Auch wenn Ettinger (2007: 902) noch zuzustimmen ist, dass ,wissenschaftlich abgesicherte Zahlenangaben zur absoluten und relativen Frequenz der Phraseme“ als ,[e]in dringendes Desiderat für die Phraseodidaktik" anzusehen ist, gibt es in diesem Bereich schon erste Forschungsansätze und -ergebnisse. Die deutschen Phraseologismen im EPHRAS-Projekt wurden auf ihre Geläufigkeit bei Muttersprachlern überprüft (cf. Jesenšek 2006; Šajanková 2005, 2005a) und eine erste Bestimmung eines Grundwortschatzes für die deutsche Phraseologie wird in Hallsteinsdóttir/Šajanková/Quasthoff (2006) als phraseologisches Optimum vorgestellt ${ }^{11}$. Für diese Bestimmung wurden Ergebnisse aus zwei Studien kombiniert ausgewertet:

1. Die erste Untersuchung enthält Frequenzdaten zu gut 5000 Phraseologismen aus einer Frequenzbestimmung deutscher Phraseologismen in den Korpora des Projekts Deutscher Wortschatz ${ }^{12}$. Die Phraseologismen wurden v.a. Wörterbüchern für Deutsch als Fremdsprache entnommen, um die Relevanz der überprüften Phraseologie sicher zu stellen.

2. In der zweiten Untersuchung (cf. Šajanková 2005, 2005a), deren Ergebnisse u.A. in das EPHRAS-Projekt eingeflossen sind (cf. Jesenšek 2006: 142), wird die Geläufigkeit von Phraseologismen bei Muttersprachlern anhand einer Fragebogenuntersuchung bestimmt. Die untersuchten Phraseologismen wurden ebenfalls aus DaF-Wörterbüchern extrahiert.

Es liegen Frequenz- und Geläufigkeitsdaten zu insgesamt 1.112 Phraseologismen aus DaFWörterbüchern und anderen DaF-relevanten Quellen vor. Die Auswertung der Daten erfolgte im Hinblick auf eine differenzierte Klassifikation der Phraseologismen, wobei das Zusammenspiel zwischen Geläufigkeit und Frequenz untersucht und die daraus folgende Relevanz für mündliche oder schriftliche Sprachverwendung bestimmt wurden (cf. Hallsteinsdóttir/Šajanková/Quasthoff 2006).

Ein wichtiges Ergebnis der Auswertung soll hier betont werden: Der Vergleich der Daten hat ergeben, dass zwischen einer sehr hohen Frequenz im Korpus und einer häufigen Einstufung als geläufig und von den Sprechern selbst verwendet eine starke Relation festgestellt wurde. Im Korpus hochfrequente Phraseologismen sind demnach ausnahmslos den muttersprachlichen Sprechern bekannt und werden auch aktiv verwendet. Dieser Befund ist

\footnotetext{
11 Auch Schmale (2009) beschäftigt sich mit der Frage nach der Auswahl von Idiomen für den Fremdsprachenunterricht auf der Grundlage von quantitativen Analysen von deutschen Talkshows. Diese Textsorte hat aber nur eine begrenzte Relevanz für einen handlungsorientierten Fremdsprachenunterricht, denn sie kommt in der realen Welt der allermeisten Sprachlerner nur rezeptiv vor, wenn überhaupt, denn die Frage nach der Angemessenheit und Relevanz der in vielen Talkshows vorkommenden sprachlichen Register sollte man sich vor dem Einsatz dieser Textsorte im Unterricht gestellt haben. Das Vorkommen von Phraseologismen in Talkshows generalisierend als eine wissenschaftlich gesicherte Erkenntnis zur Brauchbarkeit im FSU darzustellen und dazu noch als Ausgangspunkt zu verwenden, um die hier vorgestellte Untersuchung von Hallsteinsdóttir/Šajanková/Quasthoff (2006), die auf der Basis von DaF-Wörterbüchern und mit umfangreichen korpuslinguistischen und durch Fragebogenuntersuchungen abgesicherten phraseologischen Optima durchgeführt wurde, aus ihrem Kontext $\mathrm{zu}$ reißen und ohne die dahinter stehende Methodik und Materialgrundlage $\mathrm{zu}$ diskutieren als unbrauchbar darzustellen, gehört m.E. schlicht nicht zum guten wissenschaftlichen Ton.

12 www.wortschatz.uni-leipzig.de (gesehen am 20. November 2010).
} 
jedoch nicht ohne Einschränkung umkehrbar, denn es gibt durchaus geläufige und aktiv verwendete Phraseologismen, die im Korpus selten vorkommen. Da diese Phraseologismen ${ }^{13}$ offensichtlich zur phraseologischen Kompetenz der befragten Muttersprachler gehören, sollten sie - je nach Grad ihrer Geläufigkeit - in den phraseologischen Grundwortschatz aufgenommen werden (cf. Hallsteinsdóttir/Šajanková/Quasthoff 2006: 125).

Eine Einordnung der Phraseologismen in den Lernwortschatz wurde nach ihrer Relevanz für die unterschiedlichen Kompetenzen (Lesen, Schreiben, Hören, Sprechen) und die Niveaustufen vom GER in 9 verschiedene Gruppen vorgenommen. Im offenen Kernbereich des Grundwortschatzes anzusiedeln sind folgende Gruppen, deren Phraseologismen dementsprechend auf einer frühen Stufe gelernt werden sollten (cf. ausführlich in Hallsteinsdóttir/Šajanková/Quasthoff 2006: 126-128)14:

143 hochfrequente und allgemein geläufige Phraseologismen:

- $\quad$ in jmds Fußstapfen treten, alle Hände voll zu tun haben

258 mittelfrequente und allgemein geläufige Phraseologismen:

- nicht auf den Mund gefallen sein, ein Tropfen auf den heißen Stein sein

223 niedrigfrequente aber allgemein geläufige Phraseologismen:

- ins Fettnäpfchen treten, wie ein Wasserfall reden

Die Untersuchung von Hallsteinsdóttir/Šajanková/Quasthoff (2006) liefert nur eine Teilantwort darauf, welche Phraseologismen gelernt werden sollten. Die von ihnen aufgestellten Gruppen bilden

[...] einen ersten Vorschlag für den Kernbereich eines phraseologischen Optimums für Deutsch als Fremdsprache [...]. Diese Phraseologismen sollen und können allerdings kein endgültiges abgeschlossenes phraseologisches Optimum für Deutsch als Fremdsprache darstellen, denn es wurde zugegebenermaßen nur ein kleiner Ausschnitt der deutschen Phraseologie untersucht. (Hallsteinsdóttir/Šajanková/Quasthoff 2006: 129)

Es ist m.E. wichtig bei solchen Arbeiten zwischen verschiedenen Ebenen in der Phraseodidaktik $\mathrm{zu}$ unterscheiden ${ }^{15}$. Eine Bestimmung des phraseologischen Grundwortschatzes auf der Basis von Frequenz und Geläufigkeit kann nur auf der relativen oder absoluten Frequenz der einzelnen Wortschatzeinheiten im Korpus bzw. bei muttersprachlichen Sprechern basieren. Die Frequenz kann zusätzlich durch das Vorkommen in verschiedenen Textsorten differenziert werden. So entstandene Sammlungen oder Listen können nie - und dies wird auch bei Hallsteinsdóttir/Šajanková/Quasthoff (2006) nicht beabsichtigt ${ }^{16}$ - ohne didaktische Aufarbeitung als Input für das Lernen einer Sprache eingesetzt werden:

\footnotetext{
13 Einige der Phraseologismen sind vermutlich Domänen der gesprochenen Sprache zuzuordnen, um jedoch eine genaue Einordnung vornehmen zu können, müssten Untersuchungen zur Verwendung und Textsortenspezifik jedes einzelnen Phraseologismus durchgeführt werden.

14 Die 143 Phraseologismen aus der ersten Gruppe sind als Anhang zu Hallsteinsdóttir/Šajanková/Quasthoff (2006) publiziert. Die Phraseologismen aus den anderen Gruppen sind auf Anfrage bei den Autoren erhältlich.

15 Eine Erwartung des Vorkommens der in Forschungsarbeiten oder Wörterbüchern verwendeten Nennformen die Abstraktionen sind - in der Sprachverwendung sowie die Gleichstellung von Forschungsresultaten und didaktisch aufbereiteten Unterrichtsmaterialien (wie in Schmale 2009: 160, 174) deuten auf eine fehlende Unterscheidung und Differenzierung dieser Ebenen hin.

16 Schmale (2009: 153 ff., 174 ff.) zweifelt an der Repräsentativität und Validität des phraseologischen Optimum von Hallsteinsdóttir/Sajankova/Quasthoff (2006) und er übt starke Kritik an dieser und anderen „Listen von Phrasemen (...), die FS-Lerner erwerben sollen“. Bei keiner mir bekannten Arbeit zur Bestimmung des phraseologischen Grundwortschatzes wird der Anspruch erhoben, etwas anderes als Material für weitere
} 
Um die in der Liste aufgeführten Phraseologismen in Wörterbücher aufzunehmen oder im Fremdsprachenunterricht einzusetzen, ist u. a. eine ausführliche lexikographische Beschreibung, die Durchführung intersprachlicher Vergleiche und eine didaktische Aufarbeitung notwendig (Hallsteinsdóttir/Šajanková/Quasthoff 2006: 128)

Neben der Weiterentwicklung von Methodik steht eine phraseodidaktische und phraseographische Aufarbeitung der zum Grundwortschatz gehörenden Phraseologismen auf der Grundlage authentischer Verwendungskontexte und im Vergleich zur jeweiligen Muttersprache der Deutschlerner weitestgehend noch aus (cf. zur kontrastiven Vermittlung von Phraseologie z.B. Holzinger 1998 und zur phraseodidaktischen Umsetzung z.B. Jesenšek 2006). Auch Hallsteinsdóttir/Šajanková/Quasthoff sehen ihren

... Beitrag als einen ersten Schritt auf dem Weg, konkretes phraseologisches Material für den Fremdsprachenunterricht aufzuarbeiten. Eine spezifischere didaktische Aufarbeitung muss in jedem Falle noch erfolgen. (Hallsteinsdóttir/Šajanková/Quasthoff 2006: 128)

Weitere Untersuchungen sind daher notwendig zur Bestimmung, Beschreibung und Kategorisierung des phraseologischen Grundwortschatzes für Deutsch als Fremdsprache nach formalen, inhaltlichen und funktionalen Kriterien, deren Auswahl ebenfalls noch nach ihrer Relevanz für den Fremdsprachenunterricht festzulegen wäre.

Insbesondere bei Phraseologie im weiten Sinne ,ist natürlich von einer beträchtlichen Vielfalt des Gegenstandsbereichs auszugehen.“ (Lüger 1997: 88) Lüger schlägt drei größere Kategorien - Phraseolexeme, Sprichwörter und Routineformeln - vor, die methodisch „mit Blick auf die unterrichtliche Vermittlung auseinanderzuhalten“ (Lüger 1997: 88) sind.

Kritisiert wird die Arbeit von Hallsteinsdóttir/Šajanková/Quasthoff (2006) dafür, dass darin unterschiedliche Arten von Phraseologismen behandelt werden (cf. Schmale 2009). Schmale (2009: 150) bemängelt außerdem, dass in den Arbeiten von Jesenšek (2006) und Šajanková (2005, 2005a) „nicht zwischen einzelnen Phraseologismustypen“ unterschieden wird. Er bezieht sich dabei auf die Einteilung in „kompositionell interpretierbare Phraseme“ (Schmale 2009: 150: Fußnote 6) und „nicht-satzwertige Phraseologismen mit nicht-kompositioneller Bedeutung“ (Schmale 2009: 150). Trotz zahlreicher theoretisch-methodischer Ansätze zur Aufteilung der Phraseologie nach dem Grad ihrer Idiomatizität, Kompositionalität, Transparenz oder anderer semantischer Eigenschaften, steht eine objektive Beschreibungsmethodik noch aus, d.h. eine Kategorisierung kann nur auf der Basis des individuellen und somit subjektiven Sprachwissens der jeweiligen Forscher erfolgen. Unklar ist daher, inwieweit eine Unterscheidung zwischen den in der Phraseologieforschung angenommenen Kategorien der Phraseologie für den Fremdsprachenunterricht sinnvoll ist. So zeigt die Untersuchung von Hallsteinsdóttir (2001), dass solche theoretisch-methodische Einteilungen beim Verstehen fremdsprachlicher Phraseologismen wenig Relevanz besitzen, denn auch die Bedeutung von unbekannten nicht-kompositionellen Phraseologismen kann richtig konstruiert werden ${ }^{17}$. Die Rolle der einzelnen Komponenten spielt nachweislich eine nicht unbedeutende Rolle in der muttersprachlichen Sprachverarbeitung (cf. Rabanus et al. 2008). Hallsteinsdóttir (2001: 303) stellt ebenfalls einen starken „Einfluss der Bedeutung der einzelnen Komponenten auf die Konstruktion der phraseologischen Bedeutung“ fest. Die daraus abgeleitete Erkenntnis ist,

dass Phraseologismen in der Sprachverarbeitung als analysierbare Wortkombinationen behandelt werden, in denen den einzelnen Komponenten eine bestimmbare Bedeutung zugeordnet wird. Bei der Analyse dieser Bedeutung kann ein Fremdsprachler sowohl auf die in

\footnotetext{
Arbeiten zu liefern und somit ist die ganze doch recht scharfe Kritik von Schmale „auf Sand“ - und einem gravierenden Missverständnis der kritisierten Arbeiten - gebaut.

${ }^{17}$ Dies gilt auch beim Verstehen von unbekannten muttersprachlichen Phraseologismen, das noch weitestgehend unerforscht ist.
} 
der Regel dominante muttersprachliche Bedeutung als auch auf die Bedeutungen der einzelnen

Komponenten in der Fremdsprache zurückgreifen. (Hallsteinsdóttir 2001: 303-304)

Dies manifestiert sich einerseits in einer prinzipiellen Möglichkeit der kompositionellen Verarbeitung, die bei allen Phraseologismen durch ihre formale Beschaffenheit gegeben ist (cf. ausführlich dazu in Hallsteinsdóttir/Farø 2010). Andererseits schlussfolgert Hallsteinsdóttir (2001: 307), dass es in der Sprachverwendung ,eine prinzipielle Motivierbarkeit phraseologischer Bedeutungen“ gibt, „die als eine Erwartungshaltung der Sprecher aufzufassen ist." Die damit verbundene Thematik der phraseologischen Kompetenz wird im nächsten Abschnitt vertieft und in Relation zum Phänomen der Multimodalität diskutiert.

\subsection{Phraseologische Kompetenz und Multimodalität}

Dass es eine phraseologische Kompetenz gibt, wird allgemein vorausgesetzt (cf. ausführlich zu unterschiedlichen Auffassungen und Aspekten der phraseologischen Kompetenz in Hallsteinsdóttir 2001: 11 ff. und Hallsteinsdóttir 2011). Sie

besteht zunächst einmal darin, die Bedeutung von Phrasemen zu kennen und diese dann kontextangemessen verwenden zu können. Vor allem zeigt sich aber, dass ein Phrasem zum Ausgangspunkt für die Anwendung einer produktiven phraseologischen Kompetenz wird: Auf seiner Grundlage werden neue Ausdrücke gebildet. Es deutet sich damit an, dass die kompetente Verwendung von Phrasemen mehr voraussetzt als die Kenntnis der syntaktischen und semantischen Eigenschaften von Ausdrücken. [...] Phraseologische Kompetenz hat demnach auch eine sehr wichtige pragmatische Komponente. (Erhardt 2007: 258)

Die einzelnen Aspekte der phraseologischen Kompetenz, sowie die Frage danach, wie mutterund fremdsprachliche Phraseologismen in der Sprachverwendung tatsächlich verarbeitet werden und welche Faktoren das Verstehen und Verwenden von Phraseologismen auf welche Art und Weise beeinflussen, sind empirisch nur wenig untersucht worden.

Sprachlerner tendieren dazu, muttersprachliche Einheiten und Regeln beim Verstehen und in der Sprachproduktion unreflektiert in die Fremdsprache $\mathrm{zu}$ übertragen. Auch in der Phraseologie wird häufig eine meist durch die Form bedingte Symmetrie zwischen der Mutter- und der Fremdsprache vorausgesetzt. Fehler entstehen dann, wenn durch die Vorgaben der muttersprachlichen Phraseologie in der Fremdsprache fehlerhafte oder nicht existente Phraseologismen produziert werden oder wenn die muttersprachliche Bedeutung bei ähnlichen Phraseologismen (falsche Freunde, Quasisynomyme, asymmetrische Bedeutungsstrukturen) konstruiert wird (cf. Hallsteinsdóttir/Farø 2010).

In ihrer Arbeit stellt Hallsteinsdóttir ${ }^{18}$ (2001) anhand der Ergebnisse einer empirischen Untersuchung die Faktoren dar, die einen Einfluss auf das Verstehen fremdsprachlicher Phraseologismen ausüben. Im Folgenden werden ihre Ergebnisse kurz skizziert. Sie beschreibt Strategien, die in der Fremdsprache Deutsch bei der Entschlüsselung und Konstruktion phraseologischer Bedeutungen bei bekannten und unbekannten Phraseologismen angewendet werden und die somit als Komponenten der phraseologischen Kompetenz anzusehen sind. Dabei konstatiert sie einen relativ großen Einfluss der Muttersprache auf das Verstehen fremdsprachlicher Phraseologismen (cf. auch Jesenšek (2006) und Reder (2006; 2008, in diesem Heft) zu Auswirkungen der muttersprachlichen phraseologischen Kompetenz auf das Fremdsprachenlernen), denn es

wird die Hypothese bestätigt, dass die muttersprachliche phraseologische Kompetenz bewusst oder unbewusst auf fremdsprachliche Phraseologismen übertragen wird und so die Grundlage für das Verstehen fremdsprachlicher Phraseologismen bildet. Sowohl geläufige als auch

18 Online-Version unter http://www.verlagdrkovac.de/volltexte/3-8300-0435-4 (gesehen am 5. November 2010). 
unbekannte fremdsprachliche Phraseologismen, deren muttersprachlichen Äquivalente im mentalen Lexikon der VP vorhanden sind, werden durch muttersprachlichen Transfer ohne Schwierigkeiten verstanden. (Hallsteinsdóttir 2001: 300)

Der Einfluss der Muttersprache zeigt sich außerdem darin, dass bei den untersuchten falschen Freunden und den zwei wörtlich übersetzten muttersprachlichen Phraseologismen, die es im Deutschen nicht gibt, die muttersprachliche Bedeutung und ein hoher Geläufigkeitsgrad konsequent angegeben wurden. Aber nicht nur bei phraseologischer Äquivalenz ist ein muttersprachlicher Einfluss vorhanden:

Die Vorgaben des muttersprachlichen Sprachsystems bieten potentielle überindividuelle Vernetzungen des mentalen Lexikons, die durch gemeinsames lexikalisches Wissen, bevorzugte semantische Relationen und Assoziationen hervorgerufen werden. Ebenso bilden gemeinsame Lebenserfahrungen und überindividuelles Wissen über (historische) Ereignisse die Grundlage für den (intertextuellen) Bezug auf bestimmte Situationen. Ein Fremdsprachler verfügt außerdem über eine potentielle intralinguale fremdsprachliche Motivierungsbasis, sowie über vielfältige weitere interlinguale Motivierungsstrategien, die auf der Relation der jeweiligen Muttersprache zu anderen Fremdsprachen basieren. (Hallsteinsdóttir 2001: 301)

Die phraseologische Kompetenz besteht, vereinfacht ausgedrückt, aus dem Wissen über die Phraseologie. Dieses Wissen beinhaltet einerseits die geläufigen Phraseologismen, die im mentalen Lexikon enthalten sind sowie die Verbindungen, die diese Phraseologismen zu anderen Einheiten des mentalen Lexikons eingehen. Andererseits gehen Verstehensstrategien für bekannte und unbekannte Phraseologismen sowie Strategien für sowohl die normgerechte als auch die kreative Verwendung von Phraseologismen in dieses Wissen ein. Die zusammenfassende Schlussfolgerung von Hallsteinsdóttir (2001) beschreibt die Komplexität der interlingualen phraseologischen Kompetenz:

Die Ergebnisse der Tests lassen darauf schließen, dass Fremdsprachler nicht nur die muttersprachliche phraseologische Kompetenz auf die Fremdsprache übertragen, sondern auch zusätzlich eine fremdsprachliche phraseologische Kompetenz bilden, die von der muttersprachlichen Kompetenz allerdings kaum getrennt werden kann, und daher als eine Mischkompetenz anzusehen ist. Durch den muttersprachlichen Einfluss haben Fremdsprachler deutlich andere Verstehensvoraussetzungen als Muttersprachler. Dennoch ist anzunehmen, dass die in dieser Arbeit dargestellten Motivierungs- und Verstehensstrategien als solche nicht sprachenspezifisch sind, dass aber die Verwendung der Strategien durch die individuelle Sprachkompetenz, das Wissen und die Lebenserfahrungen der Sprecher sowie vorhandene Kontextinformationen und die Beschaffenheit des jeweiligen Phraseologismus bedingt wird. Die Konstruktion fremdsprachlicher Bedeutungen stellt ein komplexes Verfahren dar, das in einer Interaktion vom Textinhalt und dem eigenen Wissen der VP mit Hilfe unterschiedlicher, nicht eindeutig abgrenzbarer Verstehens- oder Motivierungsstrategien erfolgt. (Hallsteinsdóttir 2001: 306-307)

Die Arbeit von Hallsteinsdóttir gibt erste Einblicke in die phraseologische Kompetenz. Eine umfassende Erfassung und Beschreibung der konkreten Aspekte der phraseologischen Kompetenz steht jedoch noch aus, d.h. es fehlt eine ausreichende Antwort auf die Frage, was es genau bedeutet, die phraseologische Bedeutung zu kennen und anwenden zu können und was ein Sprecher können muss, um einen Phraseologismus adäquat zu verwenden oder mit Phraseologie kreativ umzugehen. Mit der Erfassung und Beschreibung von u.a. diesen Aspekten der phraseologischen Kompetenz zwecks Einbindung von Vorwissen und Verstehensstrategien der Lerner - also der muttersprachlichen phraseologischen Kompetenz in den Lernprozess könnte der Fremdsprachendidaktik eine Grundlage zur gezielten, kontrastiv verankerten Vermittlung von Phraseologismen zur Verfügung gestellt werden (cf. auch Aguado 2002; Jesenšek 2006; Reder 2006, 2008).

Im Folgenden wird beispielhaft ein Aspekt der phraseologischen Kompetenz aufgegriffen, der über die Beschreibung der Phraseologie als systemhafte Sprachzeichen hinausgeht und das 
Zusammenspiel von sprachlichen und nicht sprachlichen Kommunikationsmitteln berücksichtigt ${ }^{19}$. Dieser Aspekt wird häufig als Multimodalität bezeichnet, die als die parallele Einbeziehung von zwei oder mehr der fünf menschlichen Sinne bei einer kognitiven Tätigkeit, hier bei der Verarbeitung von Sprache, verstanden wird. Hier liegt der Fokus v.a. auf dem Aspekt der Visualisierung (cf. auch ausführlich dazu in den Beiträgen in Fix/Wellmann (2000) sowie Rentel 2005, in diesem Heft) und außerdem werden kurz einige Besonderheiten der Phraseologie in der gesprochenen Sprache aufgezeigt.

Es gilt als allgemein akzeptiert, von Bildsphären (auch: Bildlichkeit, Bildhaftigkeit, Visualisierung, Ikonizität) in Phraseologismen zu sprechen. Ihre Art und ihre Rolle im Sprachsystem und in der Sprachverwendung sind hingegen nicht vollständig aufgeklärt. Visualisierungsverfahren in der Phraseologie hängen eng mit der Relation zwischen Bedeutung (mentale Konzepte, phraseologische Bedeutung und die Bedeutungen der einzelnen Komponenten) und der äußeren Form zusammen. Die folgenden Abbildungen 1-5 zeigen unterschiedliche Arten und Komplexität von Visualisierung, bei deren Realisierung in den jeweiligen Kontexten sowie selbstverständlich auch beim Verstehen im Rezeptionsprozess auf die phraseologische Kompetenz zurückgegriffen werden muss.

Visuelle Multimodalität manifestiert sich in Abbildung 1, indem die sprachliche Form grünes Licht zur Visualisierung einer grünen Ampel verwendet wird, die freie Fahrt verspricht. Die Ampel ist in eine in der Farbe Grün gehaltenen Kulisse eingebettet, die als Rahmen für den Parteitag der politischen Partei Die Grünen dient.

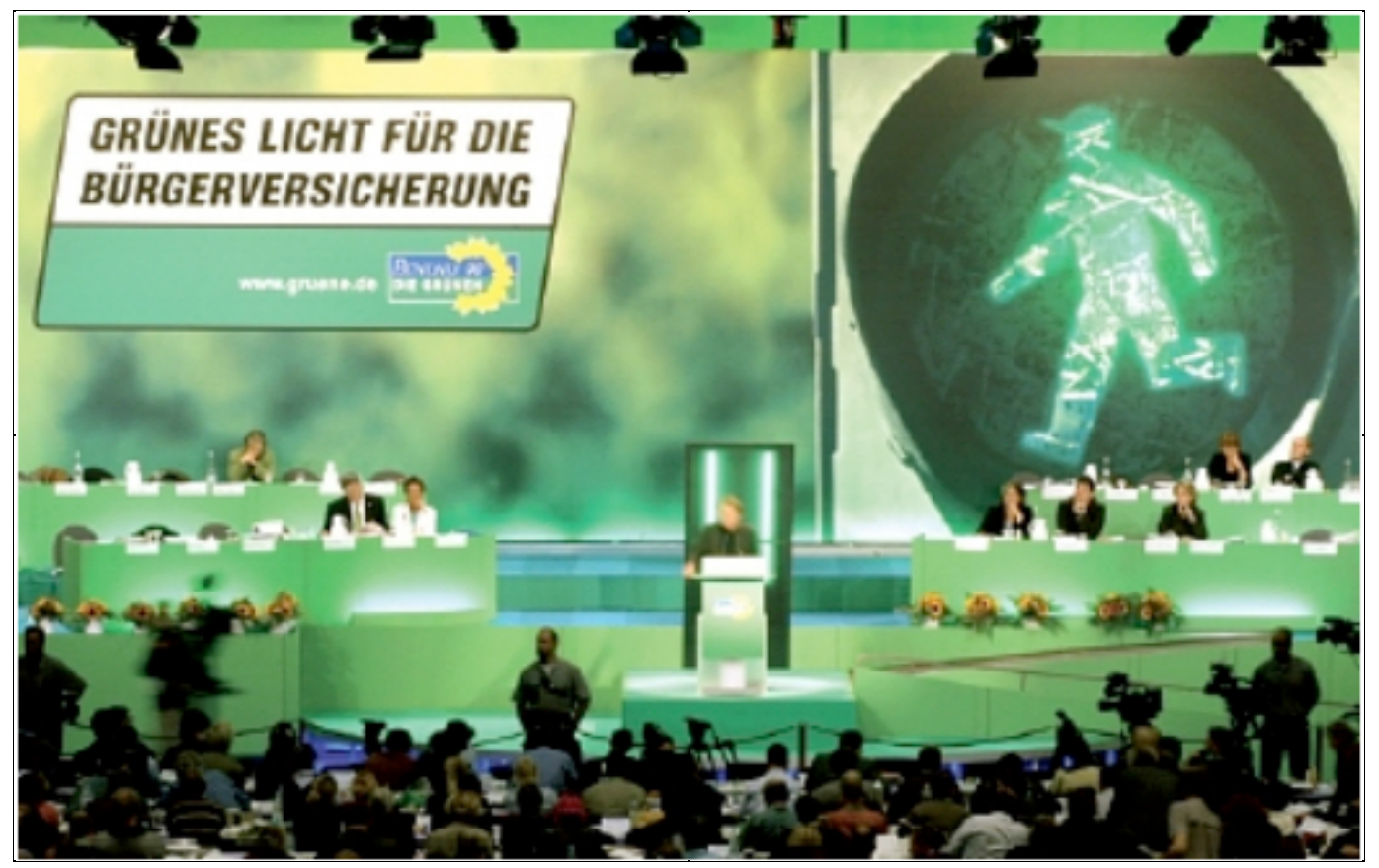

Abbildung 1: Visuelle Realisierung der sprachlichen Form von grünes Licht $^{20}$

Die Vorstellung (das mentale Konzept) einer Verkehrsampel bildet - evtl. als eine konzeptuelle Metapher - die Grundlage für andere Modifikationen dieses Phraseologismus wie in folgenden beiden Beispielen, in denen die Signalfunktionen der Farben grün, gelb und rot thematisiert werden (cf. Farø 2006 zur Thematisierung):

19 Multimodalität wird interlingual nicht einheitlich definiert, so umfasst sie in der dänischen Multimodalitätsforschung z.B. das Zusammenspiel unterschiedlicher semiotischer Ressourcen in der Kommunikation (cf. z.B. Boeriis 2008; Andersen/Boeriis 2008), die in der deutschsprachigen Forschung z.T. mit dem Begriff Multicodierung erfasst werden.

${ }^{20} \mathrm{http}: / /$ www.deutschesaerzteblatt.de/v4/archiv/artikel.asp?id=43686 (gesehen am 2. Oktober 2010). 
- Mal gaben demokratische Präsidenten grünes Licht, dann schalteten konservative wie zuletzt George W. Bush auf Rot ${ }^{21}$.

- Denn wie berichtet, hat die Bundeswettbewerbsbehörde zwar die (nicht anmeldungspflichtige) Finanzbeteiligung erlaubt, aber für Kooperationen zwischen den beiden Handelsketten ,gelbes Licht" gegeben: Das heißt, dass die Zusammenarbeit laufend beobachtet wird ${ }^{22}$.

Aber auch umgekehrt zur Abbildung 1 gilt, dass die visuelle Form zum Phraseologismus führen kann, wie in Abbildung 2, in der die rote Farbe der Zahlen, die Verluste anzeigen, den Sprecher zur Bedeutung des Phraseologismus rote Zahlen schreiben führt.

Umsatz- und Gewinnentwicklung bei Apple in US-Dollar

(Das Geschäftsjahr beginnt jeweils zum Oktober.)

\begin{tabular}{l|l|l|}
\hline Quartal & Umsatz & $\begin{array}{l}\text { Nettogewinn/ } \\
\text {-verlust }\end{array}$ \\
\hline $1 / 01$ & 1.000 Mio. & $-195 \mathrm{Mio}$. \\
\hline $2 / 01$ & $1.430 \mathrm{Mio}$. & 40 Mio. \\
\hline $3 / 01$ & 1.480 Mio. & 61 Mio. \\
\hline $4 / 01$ & 1.450 Mio. & 66 Mio. \\
\hline $1 / 02$ & 1.380 Mio. & 38 Mio. \\
\hline $2 / 02$ & 1.500 Mio. & 40 Mio. \\
\hline $3 / 02$ & 1.430 Mio. & 32 Mio. \\
\hline $4 / 02$ & 1.440 Mio. & -45 Mio. \\
\hline $1 / 03$ & 1.470 Mio. & -8 Mio. \\
\hline $2 / 03$ & 1.475 Mio. & 14 Mio. \\
\hline
\end{tabular}

Abbildung 2: Visualisierung des Phraseologismus rote Zahlen schreiben über seine Bedeutung und die Farbe $\operatorname{Rot}^{23}$

Ein komplexes Beispiel findet man in Abbildung 3, in der der Phraseologismus etw. auf den Punkt bringen thematisiert wird. Sowohl die wörtliche Bedeutung der Komponente Punkt als auch die u.A im DUDEN kodifizierte Bedeutung, etw. präzise zum Ausdruck bringen“ spielen eine zentrale Rolle in der Gestaltung der Werbung für einen Drucker:

- Die Visualisierung der Komponente Punkt als gedruckter Punkt (Pixel) bezieht sich auf die Visualisierung der Farbtiefe des Druckergebnisses des beworbenen Druckers. Somit entsteht eine Ambiguität, bei der dem Leser die Aktivierung des Phraseologismus überlassen wird, denn gezeigt wird nur eine vergrößerte Abbildung eines Pixels: Wir bringen mehr Farbe auf den

- Es erfolgt eine Thematisierung über die wörtliche Bedeutung als Farbpunkt (DOT)/Pixel bei Laserdruckern mit der Formulierung Nicht die bloße Anzahl der Punkte bestimmt das Druckergebnis.

\footnotetext{
${ }^{21} \mathrm{http} / / / w w w . z e i t . d e / 2002 / 38 /$ Stammzellen_von_der_Nationalbank (gesehen am 29. September 2010).

$22 \mathrm{http} / / /$ diepresse.com/home/wirtschaft/economist/348583/index.do (gesehen am 29. September 2010).

${ }^{23}$ Heise online (Tabelle verkürzt): http://www.heise.de/newsticker/meldung/61682 (gesehen am 29. September 2010).
} 
- Die Überschrift wird mit Verbalisierung des Idioms: Farbintelligenz auf den Punkt gebracht am Ende des Textes wieder mehrdeutig aufgenommen, wobei eine Interpretation dem Leser überlassen wird.

- Möglicherweise enthält die Anzeige auch noch eine Anspielung auf die Bedeutung des Phraseologismus ,etwas präzise zum Ausdruck bringen', denn diese Bedeutung ist in Relation zur Werbung als mehrdeutig anzusehen, d.h. als etwas Gedrucktes und z.B. als künstlerische Gestaltung, Ausdrucksweise etc. Diese Mehrdeutigkeit ist ebenfalls in weiteren Formulierungen der Anzeige zu sehen:

- Konica Minolta: Der Ausdruck für mehr Farbintelligenz.

- Das ist ein völlig neuer Ansatz, um exzellente Ausdrucke zu erreichen.

- Nuancenreichere Pixel für noch feinere Farbverläufe in einer ausdrucksstarken Contone-Fotoqualität mit brillanter Farbwiedergabe

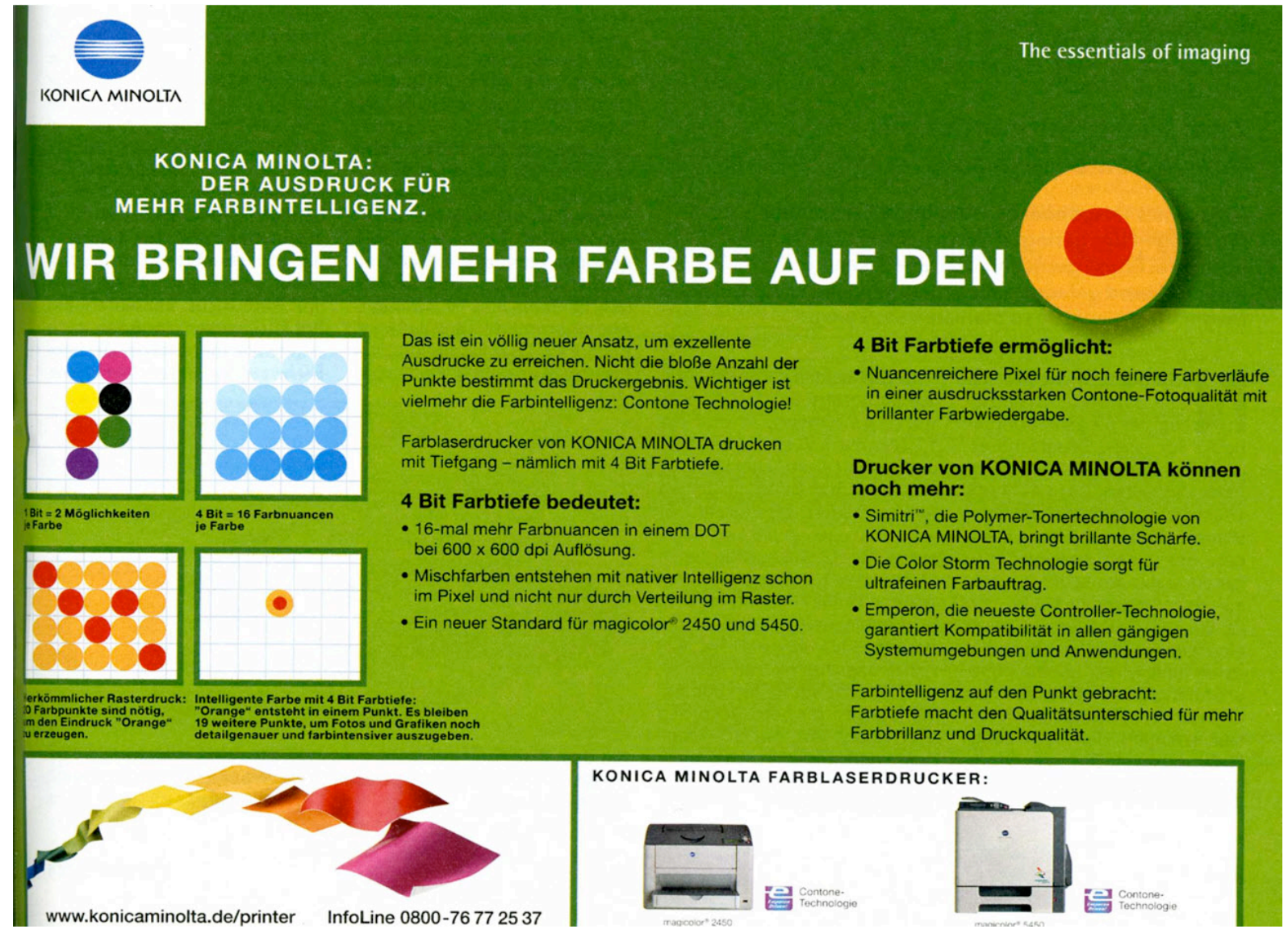

Abbildung 3: Eine multimodale Verwendung des Phraseologismus etw. zum Ausdruck bringen in einer Anzeige aus c't 4/2006, S. 93.

Mit dem Schaf Emma ${ }^{24}$ in der Hauptrolle läuft seit etwa fünf Jahren eine cross-mediale Werbekampagne der Union Investment und der Volksbank, in der mit dem Motto „Bring Deine Schäfchen ins Trockene“ bzw. „Bringen Sie Ihre Schäfchen ins Trockene“ für Zusatzrenten geworben wird. Die Realisierung der Kampagne, die regelmäßig an die beworbenen Leistungen angepasst wird, erfolgt v.a. im Internet und im Fernsehen, aber auch mit einzelnen Veranstaltungen der beteiligten Banken, wie z. B. dem

\footnotetext{
24 https://www.vrsta.de/Schaf-Emma.915.0.html?\&no_cache=1 (gesehen am 13. Juli 2010).
} 
Schäfchenschwimmwettbewerb der Volksbank Rhein-Lahn eG ${ }^{25}$. Die früheren Kampagnen benutzten außerdem weitere Medien und Gadgets wie downloadbare Handy- und Computerspiele, Klingeltöne, Hintergrundbilder, Bildschirmschoner, etc. Für das Handy wurden verschiedene Anwendungen entwickelt: mit einem Schafspiel, Klingelton und Hintergrundbild mit Schaf, die man per SMS anfordern und somit das Handy zum „Schafstall“26 machen konnte. Im Fernsehen wurden Werbespots mit Schafen gezeigt, die aus dem Regen gerettet werden (cf. die Internetseite der Kampagne aus dem Jahr 2006 in Abbildung 4 und die aktuelle Kampagne in Abbildung 5).

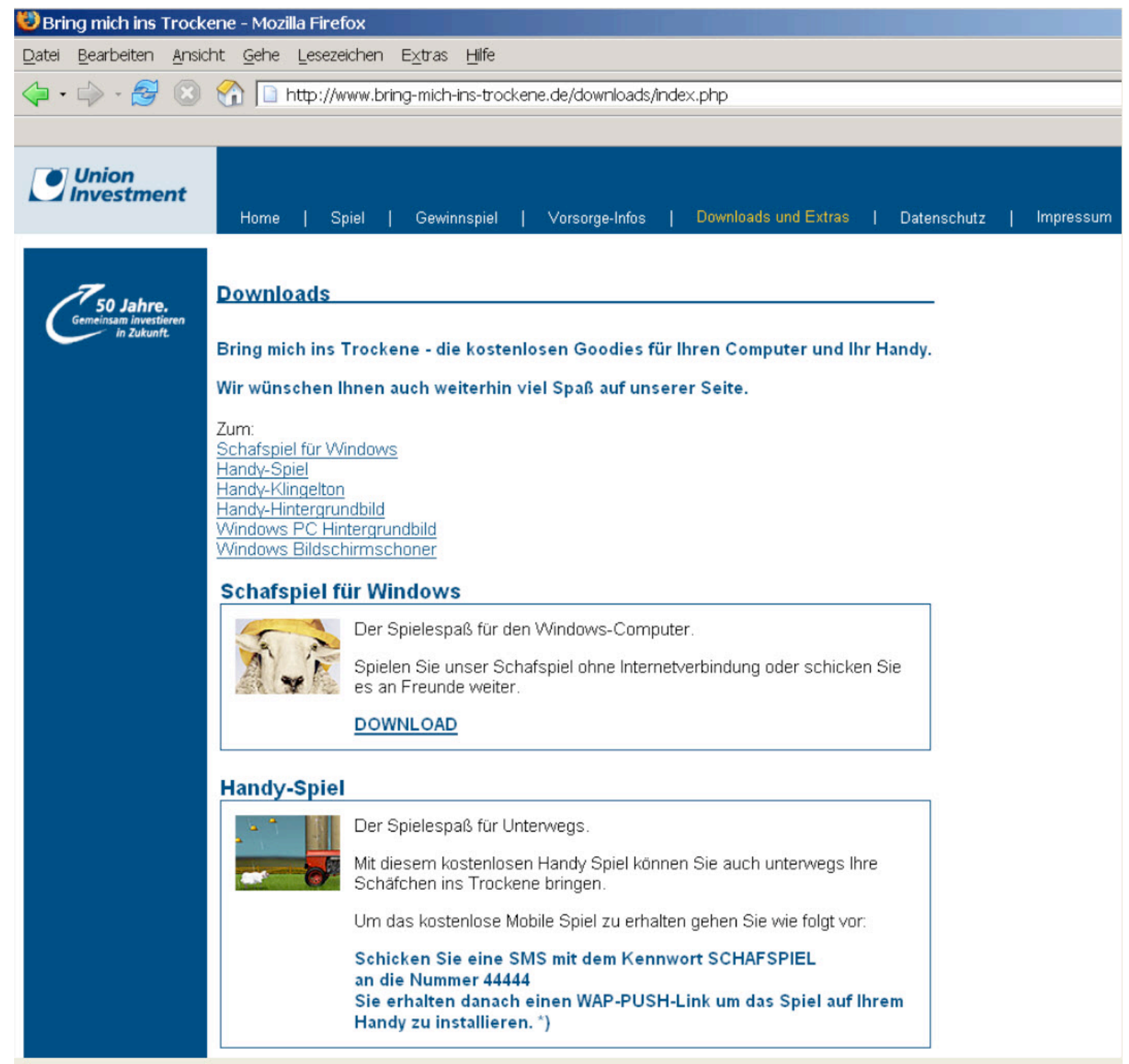

\section{Abbildung 4: Internetangebot der „Bring mich ins Trockene“ Kampagne der Union Investment 2006 (gesehen am 4. Juni 2006).}

Im Internet findet man die Kampagne unter der URL http://www.bring-mich-ins-trockene.de (gesehen am 2. Oktober 2010). In der jetzigen Version der Kampagne läuft ein Schaf über den Bildschirm mit dazugehörigen Mecker-Lauten. Farblich herrschen blau (Wasser), weiß (Wolle) und gelb (Regenbekleidung: Schafe mit Regenmütze) vor. Es gibt es ein online

\footnotetext{
25 http://www.kvl-lahnstein.de/lehnerkirmes/2008/g2008.html (gesehen am 2. Oktober 2010).

$26 \mathrm{http} / / / \mathrm{www}$.arvato-mobile.com/de/mobile-marketing/mobile-marketing-cases/union-investment.html (gesehen am 13. Juli 2010).
} 
Schäfchen-Spiel, das Schaf-Stapel-Spiel, aber die Kampagne baut v.a. auf einem Video auf, in dem etliche Schafe aus starkem Regen in ein Wohnhaus getragen werden, wonach eine Stimme aus dem Off den Zuschauer auffordert:

Jetzt mitnehmen was geht, und die Schäfchen ins Trockene bringen mit der Riester Rente vom Marktführer Union Investment.

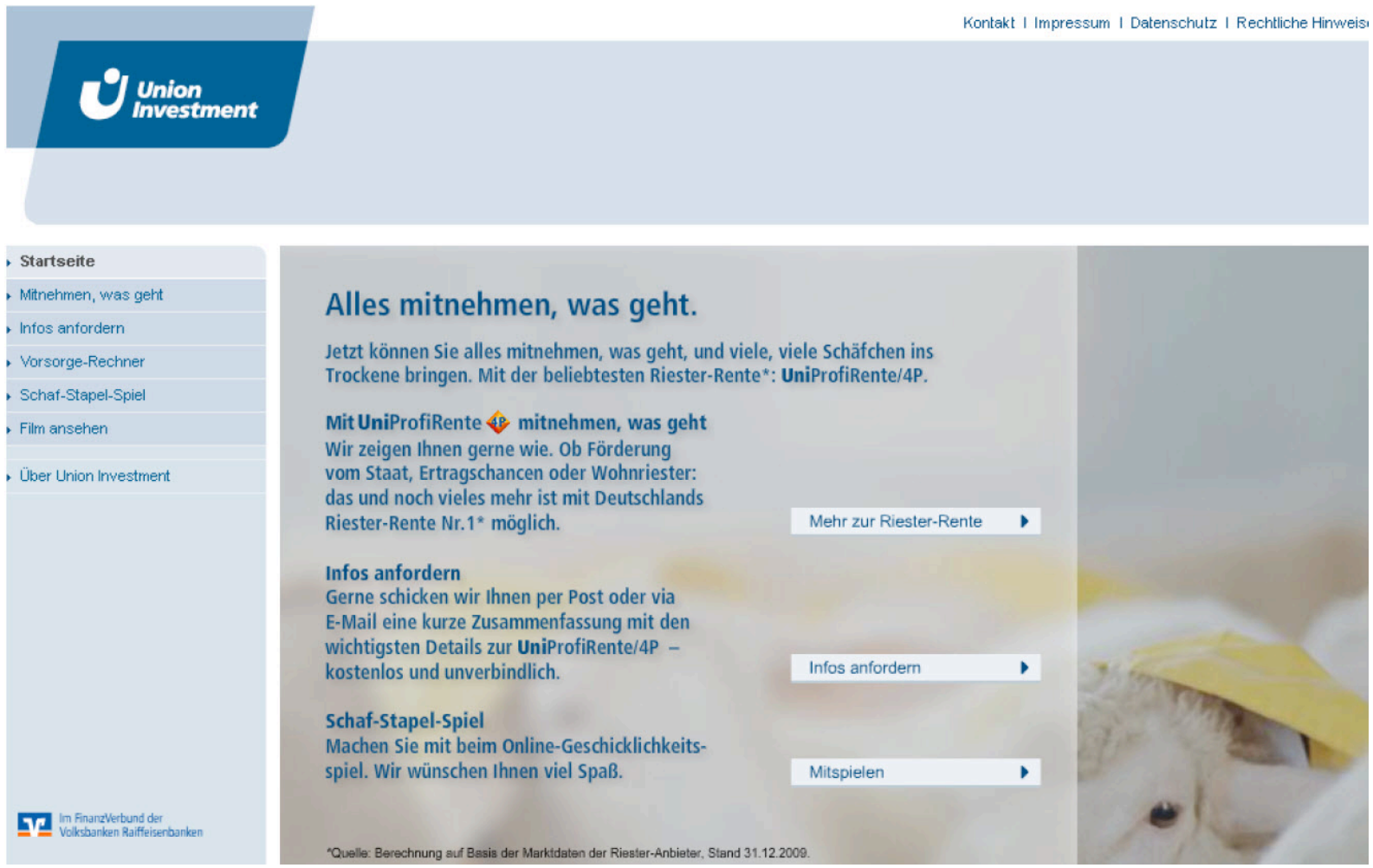

Abbildung 5: Das aktuelle Internetangebot der „Bring mich ins Trockene“ Kampagne der Union Investment $^{27}$

Das Ziel der Kampagne ist es also, ein Produkt zu verkaufen, das die finanzielle Sicherheit des Lesers im Rentenalter garantieren soll. Die komplette Kampagne baut auf dem Phraseologismus sein Schäfchen ins Trockene bringen auf. Sowohl die Formseite des Phraseologismus und der einzelnen Komponenten als auch die Bedeutung werden in der Werbung multimedial und multimodal thematisiert, indem unterschiedliche Sinneswahrnehmungen und Zeichensysteme aktiviert werden: geschriebene und gesprochene Sprache, bewegte und unbewegte Bilder, Musik, Farben, Tierlaute und Regengeräusche. Die Komponente Schäfchen wird sowohl mit Fotos und Zeichnungen als auch in Videos mit entsprechenden Lauten (Meckern) als Schaf ${ }^{28}$ visualisiert. Die Kombination ins Trockene bringen wird ebenfalls in der im Video und Spiel ausgeführten Handlung visualisiert, in der Schafe aus dem Regen an einen Trockenen Ort (Wohnung/Stall) gebracht werden. Diese Handlung wird durch die bewerbende Firma sowie den Textinhalt mit der phraseologischen Bedeutung von sein Schäfchen ins Trockene bringen ,sich Gewinn, einen Vorteil verschaffen, sichern' in Relation gesetzt, denn wenn man das beworbene Produkt kauft, so die Werbebotschaft, hat man finanzielle Sicherheit im Rentenalter.

\footnotetext{
$27 \mathrm{http} / /$ www.bring-mich-ins-trockene.de/\#/startseite/ (gesehen am 2. Oktober 2010).

28 Cf. die Bedeutungsangabe im DWDS (www.dwds.de, gesehen am 2. Oktober 2010): ,mittelgroßes, genügsames Horntier, das wegen seiner Wolle, seines Fleisches und auch wegen seiner Milch und seines Felles als Haustier gehalten wird und in steppenhaften Hügel- und Gebirgslandschaften auch wild lebt". Interessanterweise wird diese Bedeutung beim Wort Schäfchen nicht aufgeführt, sonder nur die beiden Bedeutungen ,'die jmds. Obhut anvertrauten Menschen' und 'Schäfchenwolken'.
} 
In der Werbekampagne mit dem Schaf Emma spielt die lautliche Realisierung des strömenden Regens und der meckernden Schafe eine Rolle in der ganzheitlichen Gestaltung. Neben der Möglichkeit solcher lautlicher Realisierungen von Teilbedeutungen einzelner Komponenten eines Phraseologismus ist u.a. von Hirschfeld (1996) darauf hingewiesen worden, dass die Aussprache, insbesondere die Intonation und die Satzmelodie, einer Wortkombination darüber entscheiden kann, ob es sich um eine Verwendung eines Phraseologismus als freie Wortkombination, d.h. in einer kompositionellen freien Lesart statt der lexikalisierten phraseologischen Lesart handelt. Diese Differenzierungsmöglichkeit kann gezielt eingesetzt werden, um beim Empfänger eine bestimmte Wirkung - eine Art Aha-Erlebnis - zu erreichen. Beispiele für solche Art Verwendung von Phraseologismen findet man in vielen Liedertexten.

$\mathrm{Zu}$ Funktionen von Phraseologie in literarischen Texten - zu denen Liedertexte im weiten Sinne gehören - gibt es inzwischen einige Arbeiten (cf. Preußer in diesem Band). So zählt Bernstein (2010: 204) vier Funktionen auf, ,die durch Anwendung der phraseologischen Termini auf interpretatorische Zugänge zu den Gedichten zu verzeichnen sind“" auf:

1. Themenentfaltung;

2. Sprachsensibilisierung und Sprachkritik;

3. Komik;

4. Durchbrechen lyrischer Konventionen.

Im Bezug auf Rocktexte unterscheidet Lenk (2010: 227) vier grundlegende Typen des Gebrauchs von Phraseologismen in Rocktexten (cf.. ausführlich in Lenk 2001, 2010):

1. sparsamer Gebrauch nur weniger Phraseologismen;

2. alltagsorientierter Gebrauch, bei dem ,die Texter hauptsächlich andere Verfahren der mehr oder weniger poetischen Textproduktion“ einsetzen;

3. spielerischer Gebrauch mit (a) ,auffällige[n] Modifikationen und Kontextualisierungsverfahren“ oder (b) „Kontrastierung usueller und okkasioneller (mehrgliedriger) Metaphern“ (Lenk 2010: 227)

Text- und kommunikationsorientierter Unterricht am Beispiel authentischer Texte wird schon lange gefordert. Auch wenn literarische Texte daher eher selten zu den verwendeten Texten gehören, gibt es einzelne Arbeiten, die die didaktische Aufarbeitung von Phraseologismen in literarischen Texten behandeln (cf. Henk 2001).

Aus der großen Menge an deutscher Musik sollen hier für den phraseodidaktischen Einsatz exemplarisch die Texte von Johannes Kirchberg, den Prinzen und Peter Fox genannt werden ${ }^{29}$. Die Texte enthalten alle spielerisch gebrauchte Phraseologismen und alle vier oben genannten interpretatorischen Zugänge können im Unterricht einbezogen werden. Diese Texte wurden schon im Unterricht in mündlicher Kommunikation erprobt ${ }^{30}$. Die Lieder wurden eingebettet in die für den Unterricht thematisch vorgeschriebene Bereiche aus dem Alltagsleben (in etwa entsprechend der Niveaustufe B2) eingesetzt, denn durch ihre soziokulturelle Aktualität mit Themen aus dem alltäglichen Leben, wie Liebe, Alter, Beziehungen, Familie, Elternschaft, Umwelt, Wohnen, Verkehrsmittel, Feiern, etc. sind die Texte im

\footnotetext{
29 Weitergeben möchte ich außerdem den Hinweis auf Roger Ciceros „Das ganze Leben ist ein Zoo“ als einen möglichen Einstieg in die Behandlung der Phraseologie im Unterricht, den ich Britta Winzer-Kiontke verdanke.

30 Mündliche Kommunikation 1 ist ein Pflichtfach im 1. Semester in den an der Süddänischen Universität Odense angebotenen Studiengängen Germanistik, Organisationskommunikation und Wirtschaftsdeutsch. Das Ziel des Faches ist das Trainieren der mündlichen Sprachfertigkeit in vorgegebenen (Alltags-)Situationen sowie der Aufbau eines metasprachlichen und -kommunikativen Bewusstseins über situativ adäquates Kommunizieren.
} 
Unterricht sinnvoll einsetzbar. Sie dienen sowohl als Hörverständnisübung zur Einstimmung und Diskussionsgrundlage für die jeweiligen Alltagsthemen als auch zur metasprachlichen Sensibilisierung für die Diversität des Wortschatzes sowohl bezüglich der Form, der Bedeutungen als auch der Funktionen und Registerzugehörigkeit von Wörtern und Phraseologismen.

In den Texten von Johannes Kirchberg gehören Phraseologismen zum festen Inventar. Sie werden häufig für Wortspiele eingesetzt, in denen die Aussprache benutzt wird, um eine Art Aha-Effekt mit dem Wechsel von kompositioneller zu phraseologischer Lesart - bzw. umgekehrt im letzten Beispiel - in der Textentfaltung zu erzielen, z.B. in folgenden Zeilen aus dem Lied „Heut“ fahr“ ich Rad““31:

- Heut 'fahr' ich Rad, heut" fahr' ich Rad, heut' fahr' ich Rad-Fahrer platt

- Ich hab' die Radfahrer satt, denn jeder Radfahrer hat doch nur ein Rad, doch nur ein Rad, doch nur ein, doch nur ein Rad ab

- Ich geb' Gas, ich fahr', ich bin auf hundertachtzig, zehn Sekunden später ist's mein Tacho auch

In dem Text „Mein Fahrrad“ 32 von den Prinzen wird mit verschiedenen Bedeutungen und symbolischen Werten von Farben gespielt. Nach den Begründungen für die Ablehnung der Farben Lila und Braun wird die Angemessenheit der blauen Farbe für das Fahrrad in der letzten Zeile mittels des Phraseologismus blau sein (,betrunken sein') begründet:

\section{Mein Fahrrad ist nicht lila \\ Denn das macht mich gar nicht an \\ Es ist auch nicht braun \\ Weil ich braun nicht leiden kann \\ Nein! Ich hab's blau angestrichen \\ vom Sattel bis zum Schlauch \\ Und ich find' das äußerst passend \\ denn blau bin ich manchmal auch!}

Die Musik von Peter Fox hat inzwischen ihren Weg in den DaF-Unterricht gefunden ${ }^{33}$. Die Sprache in seinen Liedern ${ }^{34}$ bezieht sich auf den Lebensraum Großstadt und bewegt sich zwischen Szenesprache und Alltags-Slang (hier muss im Unterricht unbedingt auf die Registerzugehörigkeit hingewiesen werden), wobei Phraseologismen mit und ohne Wortspiele eingesetzt werden, so z.B. in

(1) „Haus am See“
Alles gewinnen beim Spiel mit gezinkten Karten.
Alles verlieren, Gott hat einen harten linken Haken.
Ich grabe Schätze aus im Schnee und Sand.

\footnotetext{
31 http://vids.myspace.com/index.cfm?fuseaction=vids.individual\&videoid=11747128, eine weitere Version unter http://www.youtube.com/watch?v=Ha83zU6yu-8 (gesehen am 24. September 2010). Weitere Lieder von Johannes Kirchberg unter http://www.myspace.com/johanneskirchberg (gesehen am 24. September 2010).

$32 \mathrm{Zu}$ finden unter http://www.youtube.com/watch?v=fY24qc7woW4\&feature=related (gesehen am 24. September 2010).

33 Als Beispiele dafür seien die Didaktisierungen von „Haus am See“ im Lehrbuch „Schon mal gehört? Musik für Deutschlerner“ (Buchner 2009), http://www.klett.de/sixcms/list.php?page=suche\&modul=produktdetail\&isbn=3-12-675084-2) sowie Übungen unter http://www.deutsch-als-fremdsprache.de/infodienst/2009/haus-am-see-e-daf-info-2009-10.pdf genannt, auch wenn darin Phraseologisches nicht explizit behandelt wird.

34 Auf der Internetseite von Peter Fox: http://peterfox.de/ (gesehen am 24. September 2010) unter Musik zu finden.
} 
Und Frauen rauben mir jeden Verstand!

Doch irgendwann werde ich vom Glück verfolgt.

(2) „Stadtaffen“

Von Neukölln bis rauf zum Ku'damm

macht er den Affen und ihr wollt Zugaben

Ein Primat muss keinen Beruf haben

Ein Stadtaffe muss die Stadt im Blut haben

Die Vielfältigkeit der aufgezeigten Beispiele für die multimodale Verwendung von Phraseologie deutet darauf hin, dass die phraseologische Kompetenz ein recht komplexes Phänomen sein muss. In Hallsteinsdóttir/Farø (2010) wird die Komplexität von phraseologischen Sprachzeichen als eine semiotische Komplexität aufgefasst, um das Potenzial der Phraseologie, neben der Verbalsprache auch andere Zeichensysteme in ihrer Realisierung in der Sprachverwendung zu aktivieren. Um aber die Komplexität genau zu erfassen wäre als erster Schritt eine zeichentheoretische Einordnung der Multimodalität ${ }^{35}$ in der Phraseologie notwendig, denn bei einer bilateraler Zeichenauffassung ist unklar, ob sich die Multimodalität in der Inhalts- oder der Ausdrucksseite manifestiert. Die Beispiele in Abbildungen 6 und 7 lassen außerdem vermuten, dass sich bei der Multimodalität bzw. der semiotischen Komplexität nicht unbedingt um phraseologiespezifische Phänomene handelt, denn komplexe Wörter wie Mäusekino und sogar auch Simplizia wie Thun können zur Gestaltung von Bildsphären beitragen, die Visualisierungen ermöglichen (cf. Abbildung 6 und 7):

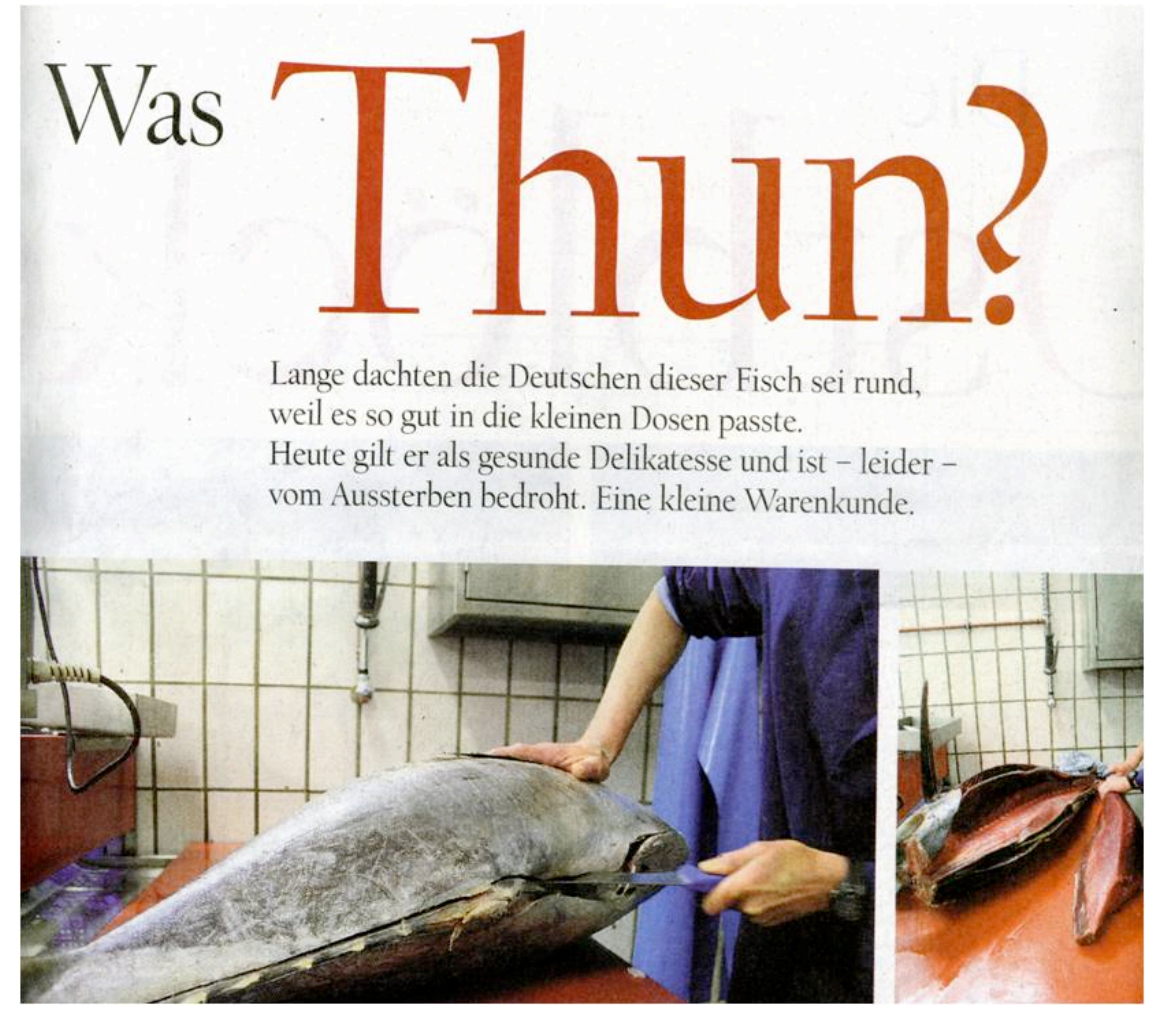

Abbildung 6: Visualisierung der Farbe des Thunfisches im Wort Thun in einer Überschrift in Der Tagesspiegel 22. Mai $2006^{36}$.

\footnotetext{
${ }^{35}$ Grundlegendes zur Semiotik gibt es u.a. in Posner/Robering/Sebeok (1997-2003).

36 Text (ohne Bilder) online: http://www.tagesspiegel.de/zeitung/was-thun/713410.html (gesehen am 2. Oktober 2010).
} 


\section{Mäusekino}

\section{Grafisches Status-Display am USB-Port}

Ein zusätzliches Minimal-Display am PC ist mehr als nur ein Gimmick für verspielte Case-Modder: Auch bei ausgeschaltetem Hauptmonitor informiert es über eingegangene E-Mails, das gerade abgespielte MP3-Musikstück oder den aktuellen Netzwerk-Traffic. Unser Projekt wartet sogar mit einem grafikfähigen Display auf, das die Informationen deutlich anschaulicher darstellen kann als die bekannten Text-Anzeigen.

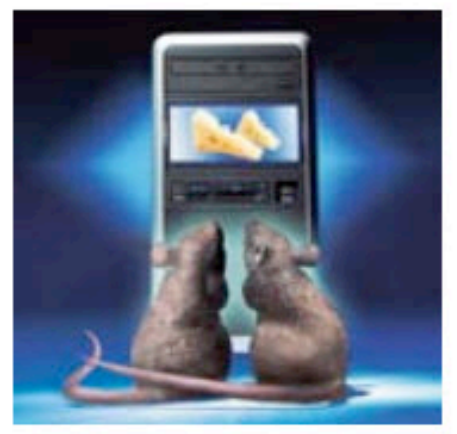

\section{Schaltungen, die ein mehrzeiliges Text-Display als} Statusanzeige zum Beispiel mit der PC-Druckerschnittstelle verbinden, kursieren seit Jahren im Internet; im ZubehörHandel erfreuen sich derartige Displays, oft aufgewertet mit einem USB-Interface, großer Beliebtheit. Bei ServerRechnern beispielsweise gehört ein kleines Display, das Traffic, Fehlermeldungen und Speicherbedarf anzeigt, durchweg zum guten Ton - der Admin erkennt dann auch ohne angeschlossenen Monitor, wie es dem Server gerade geht. Ein sicher ebenso häufiger Anwendungsfall ist der Multimedia-PC, der eine solche Anzeige als alternatives User-Interface bereithält.

\section{Abbildung 7: Visualisierung des Kompositum Mäusekino in der Computerzeitschrift c't 4/2006: 224 ${ }^{37}$.}

Um herauszufinden, inwieweit es sich beim Verstehen und Verwenden von Multimodalität um allgemeine Fähigkeiten zum Kommunizieren handelt und welche Aspekte Bestandteile einer speziellen phraseologischen Kompetenz sind, und somit in der Phraseodidaktik (sofern überhaupt noch von einer speziellen Phraseodidaktik gesprochen werden sollte) berücksichtigt werden müssen, ist noch weitere Forschung erforderlich. Außerdem kann festgestellt werden, dass noch Forschungsarbeit zur Literatur und Multimodalität im Allgemeinen und der Rolle der Phraseologie in Texten sowie der Beschaffenheit der phraseologischen Multimodalität im Besonderen notwendig ist, um eine zielgerichtete phraseodidaktische Aufarbeitung zu erleichtern.

\section{Perspektiven: Aktuelle Forschungsfragen der Phraseodidaktik}

Abgeschlossen wird dieser einleitende Beitrag mit einer Zusammenfassung von einigen aktuellen Forschungsfragen der deutschsprachigen Phraseodidaktik. Diese wurden z.T. im Abschnitt 2 erwähnt, teils werden sie in den anderen Beiträgen des Themenheftes aufgenommen und diskutiert und z.T. handelt es sich um noch offene Forschungsfragen und phraseodidaktische Desiderate. Die weiteren Beiträge ${ }^{38}$ dieses Themenheftes konzentrieren sich um verschiedene Aspekte der Phraseodidaktik, wobei sowohl Phraseologie in Lernerwörterbüchern und in Lehrwerken, die Entwicklung von phraseodidaktischen Lehr-

\footnotetext{
${ }^{37} \mathrm{http}: / / w w w . h e i s e . d e / c t / a r t i k e l / M a e u s e k i n o-290316 . h t m l$ ?view=print (gesehen am 24. September 2010).

38 Ein Teil der Beiträge sind stark überarbeitete Versionen der Vorträge der Sektion Phraseologie auf der IDT in Jena/Weimar 2009. Weitere IDT-Beiträge sowie eine Linksammlung zur Phraseodidaktik sind auf der Webseite der Sektion H6: Phraseologie unter http://idt2009h6.wordpress.com/ (gesehen am 21. November 2010) zu finden.
} 
und Lernmaterialien als auch theoretische Grundlagenforschung und aktuelle Forschungsprojekte diskutiert werden.

Didaktische Arbeiten in der Phraseologie können übergeordnet aufgeteilt werden in folgende Bereiche (cf. auch Hallsteinsdóttir/Farø 2010: 151-152):

1. Die Auswahl der zu vermittelnden Phraseologie für den Unterricht Deutsch als Fremdsprache ist eine wichtige Grundlage für die Erstellung von Lehrmaterial und Lernerwörterbüchern (cf. Bergerová 2009; Durčo 2001; Hallsteinsdóttir/Šajanková/Quasthoff 2006; Hessky 1992). Dabei handelt es sich u.A. um:

- Generelle Untersuchungen zum Status und Funktionen der Phraseologie im Wortschatz.

- Kriterien und Bedingungen für die Auswahl von Phraseologismen für den muttersprachlichen und den fremdsprachlichen Unterricht, d.h. für Lernerwörterbücher und Lehrwerke in der Muttersprache und in der Fremdsprache.

- Entwicklung von datengeleiteten Methoden für die Auffindung hoch-frequenter Phraseologismen im Korpus (cf. Quasthoff/Schmidt/Hallsteinsdóttir 2010). In seinem Beitrag „Kollokationen im Lernerwörterbuch - Anspruch und Wirklichkeit“ beschreibt Jupp Möhring (Leipzig) ein Verfahren zur Identifikation von Kollokationen in Korpora. Die so gefundenen Kollokationen verwendet er dann, um ausgewählte Lernerwörterbücher für $\mathrm{DaF}$ auf das Vorkommen von Kollokationen zu überprüfen. Dieser neuartige Ansatz zeigt eine Möglichkeit, wie die Relevanz von Phraseologismen in der Sprachverwendung festgestellt und verwendet werden kann, um Lehr- und Lernmaterialien zu validieren.

2. Die Beschreibung von Phraseologismen in der Sprachverwendung, u.a. im Hinblick auf ihre "Gebräuchlichkeit, Adressatenbezug und Textsortenspezifik" (Korhonen/Wotjak 2001: 232), ist eine wichtige Grundlage für die phraseodidaktische Aufarbeitung. Die Festlegung eines Kontextes, die Darstellung in Wörterbüchern und Lehrwerken sowie die Einbettung in eine Unterrichtssituation sollte auf dieser Grundlage erfolgen. Zu diesem Bereich gehört die Aufarbeitung des Zusammenspiels zwischen Phraseologie und der Multimodalität oder anderen nonverbalen Phänomenen in der Phraseodidaktik, u.A.:

- Die Entwicklung von Beschreibungsansätzen für Phraseologie und Multimodalität im Sprachgebrauch (z.B. Bilder, Videos, Musik, Gestik \& Mimik, Intonation) und Erfassung der daraus resultierenden Konsequenzen für das Lehren und Lernen von Phraseologismen.

- Die Umsetzung der Gestaltungsmöglichkeiten der Multimodalität in der Phraseologie in ein phraseodidaktisches Konzept thematisiert Nadine Rentel (Wien). In ihrem Beitrag „Die Didaktisierung von Phraseologismen im DaF-Unterricht anhand multimodaler Texte" geht es um phraseologische Multimodalität und textsortenspezifische Aspekte von Phraseologie am Beispiel konkreter Unterrichtsbeispiele.

3. Die Entwicklung phraseodidaktischer Unterrichtsmaterialien und die Methoden der Vermittlung von Phraseologie im Unterricht, u.a. auf der Basis von Resultaten aus der Phraseologieforschung (cf. v.a. Bergerová 2009; Ettinger 1998; Hessky 1997; Jesenšek 2006; Kühn 1992, 1994). Dies bezieht sich v.A. auf die didaktische Aufarbeitung und Integration der Phraseologie in Unterrichtsmaterialien und Wörterbüchern, wobei u.a. folgende Aspekte eine Rolle spielen:

- Die Lexikografische Aufarbeitung von Phraseologismen und die Einbeziehung der Phraseologie in die Wörterbuchbenutzungsforschung. Zwei Beiträge des Themenheftes lassen sich diesem Bereich zuordnen: Vida Jesenšek (Maribor) beschreibt in ihrem 
Beitrag „Sprichwörter im Wörterbuch“ die lexikografische Behandlung von Sprichwörtern am Beispiel der Konzeption und Entstehung der mehrsprachigen Sprichwortdatenbank SprichWort ${ }^{39}$. Im Beitrag „Anregungen zur Darstellung von Routineformeln in einsprachigen Lernerwörterbüchern für $\mathrm{DaF}^{\text {“ }}$ von Silke Lipinski (Leipzig) geht es um Lösungsmöglichkeiten für lexikografische Herausforderungen wie Auswahl, Auffindbarkeit und lexikografische Informationen bei Routineformeln.

- Für die kontextuell adäquate Integration der Phraseologie in Lehrwerken sind Untersuchungen zur Textspezifik und Funktionen von Phraseologismen notwendig. Ulrike Preußer (Bielefeld) untersucht die textgestaltenden Funktionen von Phraseologismen in Brigitte Biermanns Roman Engel haben keinen Hunger. Mit ihrem Beitrag „Phraseologismen in problemorientierter weiblicher Adoleszensliteratur - ein Beispiel“" liefert einen Beitrag zur Erforschung von Phraseologie in literarischen Texten. Hierbei handelt es sich um phraseologische Grundlagenforschung, die eine Voraussetzung der didaktischen Aufarbeitung ist.

- Die Erarbeitung multimedialer und multimodaler Unterrichtsmaterialien ${ }^{40}$ gehört zu den neueren Ansätzen der Phraseodidaktik. Gleich zwei Beiträge problematisieren den phraseodidaktischen Zugang dazu und stellen Lösungen in Form von fertig entwickelten und frei zugänglichen Lehr- und Lernmaterialien vor. Nataša Kralj und Brigita Kacjan (Maribor) geben in ihrem Beitrag „Phraseologieunterricht in der Zeit der neueren Lernmedien" Beispiele für die Nutzung von EPHRAS. Hana Bergerová (Ústí nad Labem) verbindet die Beschreibung von Anwendungsmöglichkeiten für ihr online verfügbares Unterrichtsmaterial im Beitrag „Zum Lehren und Lernen von Phraseologismen im DaF-Studium. Überlegungen $\mathrm{zu}$ Inhalten und Methoden ihrer Vermittlung anhand eines Unterrichtsmodells“ mit einem Plädoyer für die systematische Behandlung der Phraseologie im universitären Unterricht. Eng mit den in diesen beiden Beiträgen beschriebenen Materialien und Methoden hängt die Entwicklung und Umsetzung von Konzepten für autonomes Lernen von Phraseologismen ${ }^{41}$, die noch in den Anfängen steckt.

4. Die Erfassung und Beschreibung der phraseologischen Kompetenz, d.h. die durch die Sprachkompetenz gegebenen mutter- und fremdsprachlichen Voraussetzungen der Lerner (vgl. Dobrovol'skij 1995; Hallsteinsdóttir 2001; Hessky 1987; Reder 2006, 2008) ist erwünschenswert, um die Phraseodidaktik an die Voraussetzungen der Lerner anpassen zu können, wobei "auch sprachenpaarbezogene Aspekte berücksichtigt werden" (Korhonen/Wotjak 2001: 232) sollten. Darunter wird u.a. Folgendes erfasst:

- Die Entwicklung von Methoden der Erfassung und Beschreibung der mutter- und fremdsprachlichen phraseologischen Kompetenz.

- Die Verifizierung von aktuellen Auffassungen zur Rolle der muttersprachlichen phraseologischen Kompetenz beim Fremdsprachenlernen.

- Die Identifizierung und Beschreibung spezieller Problemfelder im Bezug auf die phraseologische Kompetenz in der Muttersprache und in der Fremdsprache.

\footnotetext{
39 http://www.sprichwort-plattform.org/sp/Sprichwort-Plattform (gesehen am 28. November 2010).

40 Als Beispiele für gelungene multimediale Lehrmaterialien sind z.B Hana Bergerovàs Multimediales Unterrichtsmaterial zur deutschen Phraseologie (http://frazeologie.ujepurkyne.com/index2.htm gesehen am 14. Juli 2010) und EPHRAS zu (http://demo.ephras.org/ gesehen am 14. Juli 2010) zu nennen.

${ }^{41}$ Beispielsweise Stefan Ettingers Arbeitsblatt zur Erschließung von Phraseologismen im Text und dem Anlegen einer eigenen Phraseologiesammlung (http://www.buske.de/download/Ettinger_Nunes/Arbeitsblatt_Port-RW.pdf - auch unter http://www .ettinger-phraseologie.de/pages/vorwort/vorwort5.php gesehen am 14. Juli 2010)
} 
- Die Aufarbeitung interlingualer und interkultureller Unterschiede und Gemeinsamkeiten in der Phraseologie.

- Untersuchungen zur Sprachkompetenz von bestimmten Lernergruppen, um einerseits durch die Erfassung der muttersprachlichen Voraussetzungen eine gezielte Förderung und Vermeidung von Fehlern und andererseits die Einbeziehung vorhandener Verstehens- und Verarbeitungsstrategien in das Lehren und Lernen zu ermöglichen.

- Die Untersuchung des Einflusses der phraseologischen Kompetenz in der Entwicklung einer Übersetzungskompetenz.

- Die Rolle von Motiviertheit bzw. Motivierbarkeit von Phraseologismen im Fremdsprachenerwerb wird im Beitrag von Tamás Kispál (Szeged): „Die kognitivmetaphorische Motiviertheit beim Erlernen von Idiomen am Beispiel eines Aufgabenblattes“ untersucht. Auf der Grundlage der kognitiven Metapherntheorie entwickelt er ein Aufgabenblatt, in dem die metaphorische Motiviertheit von Idiomen in den Lernprozess einbezogen wird.

5. Sprachenpaarbezogene Aspekte (cf. Korhonen/Wotjak 2001: 232) im Sprachsystem, in der Sprachverwendung und als ein Teil der Sprachkompetenz (Hallsteinsdóttir/Farø 2010) sollten in Form von kultur- und sprachvergleichende Problemstellungen und Konsequenzen soweit möglich einbezogen werden, denn der starke Einfluss der Form von muttersprachlichen Phraseologismen im Fremdsprachenlernen macht den Vergleich der Ausdrucksseite der Phraseologie (z.B. Komponentenstruktur, Ikonographie, morpho-syntaktische Eigenschaften, intrasprachlich formbedingte Wortschatzrelationen) $\mathrm{zu}$ einer wichtigen Grundlage der Phraseodidaktik.

- Anna Reder: (Pécs) diskutiert in ihrem Beitrag „Kollokationskonzepte und ihre mögliche Umsetzung in der Didaktik“ unterschiedliche linguistische Auffassungen von Kollokationen mit dem Ziel linguistische Erkenntnisse für das Fremdsprachenlernen benutzbar zu machen. Diese setzt sie in Beziehung zu eigenen Erfahrungen und Konzepten für die Vermittlung von Kollokationen, wobei sie sprachenpaarbezogene Aspekte und der Einfluss der Muttersprache in den Mittelpunkt stellt.

- Zur phraseologischen Grundlagenforschung gehört der interlingual orientierte Beitrag von Milote Sadiku (Prishtina). Sie illustriert anhand zahlreicher Beispiele mögliche Äquivalenzbeziehungen in ihrem Beitrag „Deutsche und albanische Phraseologismen mit Tierbezeichnungen im Kontrast“".

„Phraseologismen sind ganz normale Wortschatzeinheiten” ist der erste Satz eines Themenheftes von Linguistik online zu neuen theoretischen und methodischen Ansätzen in der Phraseologieforschung (Hallsteinsdóttir/Farø 2006: 3). Diese Auffassung, die hier ebenfalls den Ausgangspunkt bildet,

erfordert einen holistisch-integrativen Zugang, in dem auch Beziehungen von Phraseologismen zu anderen Sprachzeichen erfasst werden können; eine umfassende Erforschung und Beschreibung der Phraseologie kann nur in Relation $\mathrm{zu}$ anderen sprachlichen Einheiten erfolgen. (Hallsteinsdóttir/Farø 2006: 3).

Bei vielen der in diesem und in den weiteren Beiträgen des Themenheftes diskutierten Aspekten der Phraseodidaktik handelt es sich um Phänomene, die nicht unbedingt phraseologiespezifisch ${ }^{42}$ sind, die jedoch im Bezug auf die Phraseodidaktik bisher nicht

\footnotetext{
42 Dem Gutachter dieses einleitenden Beitrags gebührt ein großer Dank, nicht zuletzt für die vielen Hinweise darauf, dass viele der besprochenen Aspekte eben nicht phraseologiespezifisch sind, sondern im Spracherwerb generell zutreffen. An dieser Stelle möchten sich die Herausgeber bei den anonymen Gutachtern von Linguistik online für die vielen wertvollen Kommentare und Verbesserungsvorschläge danken.
} 
genügend erforscht und beschrieben oder für den Sprachunterricht fruchtbar gemacht wurden. Das Ziel der Herausgeber ist folglich nicht eine Abschottung der Phraseodidaktik innerhalb des Sprachdidaktik, sondern vielmehr das Vorantreiben ihrer Integration in allen Bereichen des Spracherwerbs. Wir hoffen, dass die Beiträge dieses Themenheftes für Theoretiker wie Praktiker, Linguisten wie Sprachlehrer und Lehrbuchautoren dazu vielfältige Anregungen geben können.

Abschließend möchten die Herausgeber an dieser Stelle den Autoren der weiteren Beiträge, den anonymen Gutachtern und dem Herausgeberteam von Linguistik online für ihren großen Einsatz bei der Erarbeitung dieses Themenheftes danken.

\section{Literatur}

Aguado, Karin (2002): „Formelhafte Sequenzen und ihre Funktionen für den L2-Erwerb“. Zeitschrift für Angewandte Linguistik 37: 27-49.

Andersen, Thomas Hestbæk/Boeriis, Morten (2008): "Semiotic Cereals". In: Nørgaard, Nina (ed.): Systemic Functional Linguistics in Use. Odense, University of Southern Denmark: 111-158. (= Odense Working Papers in Language and Communication 29), http://static.sdu.dk/mediafiles//Files/Om_SDU/Institutter/ISK/Forskningspublikationer/O WPLC/Nr29/Thomas\%20Hestbaek\%20Andersen\%20\%20\%20Morten\%20Boeriis.pdf (gesehen am 23. November 2010).

Babillon, Laurence (2001): „Übungstypologie zur Phraseologie des Französischen“. In: Lorenz-Bourjot, Martine/Lüger, Heinz-Helmut (eds.): Phraseologie und Phraseodidaktik. Wien: Edition Praesens: 105-124. (= Beiträge zur Fremdsprachenvermittlung, Sonderheft 4).

Baur, Rupprecht S./Chlosta, Christoph/Grzybek, Peter (1996). "Das Projekt 'SprichwörterMinima im Deutschen und Kroatischen': What is worth doing - do it well!". Muttersprache 106: 162-179.

Bergerová, Hana (2009): "Wie viel Phraseologie brauchen künftige Deutschlehrende?" In: Spáčilová, Libuše/Vaòková, Lenka (eds.): Germanistische Linguistik - die neuen Herausforderungen in Forschung und Lehre. Brno, Academicus: 71-80.

Bergerová, Hana (2010): „Multimediale Unterrichtsmaterialien für die Vermittlung von Phraseologismen. Erste Erfahrungen aus der Praxis". In: Korhonen, Jarmo/Mieder, Wolfgang/Piirainen, Elisabeth/Piñel, Rosa (eds.): EUROPHRAS 2008 Beiträge zur internationalen Phraseologiekonferenz vom 13.-16.8.2008 in Helsinki. Helsinki, Universität Helsinki: 392-398. http://www.helsinki.fi/deutsch/europhras/ep2008.pdf (gesehen am 24. September 2010).

Bergerová, Hana (o.J.): Multimediales Unterrichtsmaterial zur deutschen Phraseologie. http://frazeologie.ujepurkyne.com/index2.htm (v.A. Phraseologie in der germanistischen Ausbildung. Teil 4: Phraseologismen als ein didaktisches Phänomen. http://frazeologie.ujepurkyne.com/VS_teil04.asp) (gesehen am 28. September 2010).

Bernstein, Nils (2010): „Phraseologismen bei Nicanor Parra und Ernst Jandl““. In: Korhonen, Jarmo/Mieder, Wolfgang/Piirainen, Elisabeth/Piñel, Rosa (eds.): EUROPHRAS 2008 Beiträge zur internationalen Phraseologiekonferenz vom 13.-16.8.2008 in Helsinki. Helsinki, Universität Helsinki: 198-206. http://www.helsinki.fi/deutsch/europhras/ep2008.pdf (gesehen am 24. September 2010).

Boeriis, Morten (2008): “A Mode Instantation Model”. In: Nørgaard, Nina (ed.): Systemic Functional Linguistics in Use. Odense, University of Southern Denmark: 238-250. (= Odense Working Papers in Language and Communication 29). http://static.sdu.dk/mediafiles//Files/Om_SDU/Institutter/ISK/Forskningspublikationer/O WPLC/Nr29/Morten\%20Boeriis\%20\%20A\%20Mode\%20Instantiation\%20Model.pdf (gesehen am 24. November 2010). 
Buchner, Holm (2009): Schon mal Gehört? Musik für Deutschlerner. Stuttgart: Ernst Klett Verlag.

Burger, Harald (2007): „Phraseologie: Objektbereich, Terminologie und Forschungsschwerpunkte“. In: Burger, Harald/Dobrovol'skij, Dmitrij/Kühn, Peter/Norrick, Neal R. (eds.): Phraseologie: Ein internationales Handbuch der zeitgenössischen Forschung/Phraseology. An International Handbook of Contemporary Research. Berlin, NewYork, Walter de Gruyter: 1-10. (=Handbücher zur Sprach- und Kommunikationswissenschaft/Handbooks of Linguistics and Communication Science 28.128.2).

Burger, Harald (unter Mitarbeit von Harald Jaksche) (1973): Idiomatik des Deutschen. Tübingen: Max Niemeyer Verlag.

Daniels, Karlheinz (1985): "Idiomatische Kompetenz” in der Zielsprache Deutsch. Voraussetzungen, Möglichkeiten, Folgerungen“. Wirkendes Wort 2: 145-157.

Daniels, Karlheinz/Pommerin, Gabriele (1979): „Die Rolle sprachlicher Schematismen im Deutschunterricht für ausländische Kinder“. Die Neueren Sprachen 78/6: 572-586.

Durčo, Peter (2001): „Bekanntheit, Häufigkeit und lexikographische Erfassung von

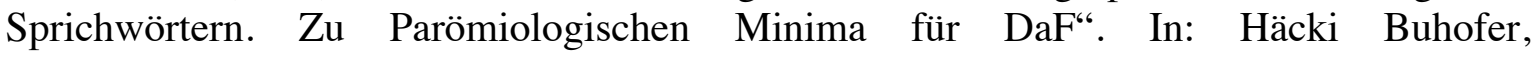
Annelies/Burger, Harald/Gautier, Laurent (eds): Phraseologiae Amor: Aspekte europäischer Phraseologie. Baltmannsweiler: Schneider Verlag Hohengehren: 99-106.

Eismann, Wolfgang (1979): „Phraseologie und Russischunterricht in der Sowjetunion“. Die Neueren Sprachen 78/6: 555-572.

Erhardt, Claus (2007): „Phraseme in der Jugendsprache“. In: Burger, Harald/Dobrovol'skij, Dmitrij/Kühn, Peter/Norrick, Neal R. (eds.): Phraseologie: Ein internationales Handbuch der zeitgenössischen Forschung/Phraseology. An International Handbook of Contemporary Research. Berlin, NewYork, Walter de Gruyter: 253-264. (=Handbücher zur Sprach- und Kommunikationswissenschaft/ Handbooks of Linguistics and Communication Science 28.1-28.2).

Ettinger, Stefan (1998): "Einige Überlegungen zur Phraseodidaktik". In: Eismann, Wolfgang (ed.): EUROPHRAS 95. Europäische Phraseologie im Vergleich: Gemeinsames Erbe und kulturelle Vielfalt. Bochum, Brockmeyer: 201-217. (= Studien zur Phraseologie und Parömiologie 15).

Ettinger, Stefan (2001): „Vom Lehrbuch zum autonomen Lernen. Skizze eines phraseologischen Grundkurses für Französisch“. In: Lorenz-Bourjot, Martine/Lüger, Heinz-Helmut (eds.): Phraseologie und Phraseodidaktik. Wien, Edition Praesens: 87-104. (= Beiträge zur Fremdsprachenvermittlung, Sonderheft 4).

Ettinger, Stefan (2007): „Phraseme im Fremdsprachenunterricht“. In: Burger, Harald/Dobrovol'skij, Dmitrij/Kühn, Peter/Norrick, Neal R. (eds.): Phraseologie: Ein internationales Handbuch der zeitgenössischen Forschung/Phraseology. An International Handbook of Contemporary Research. Berlin, NewYork, Walter de Gruyter: 893-908. (=Handbücher zur Sprach- und Kommunikationswissenschaft/ Handbooks of Linguistics and Communication Science 28.1-28.2).

Farø, Ken (2006): Idiomatizität - Ikonizität - Arbitrarität. Beitrag zu einer funktionalistischen Theorie der Idiomäquivalenz. Kopenhagen: nicht publ. Doktorarbeit an der Universität Kopenhagen.

Fix, Ulla/Wellmann, Hans (2000) (eds.): Bild im Text - Text und Bild. Heidelberg: C. Winter. Fleischer, Wolfgang $(1997)^{2}$ : Phraseologie der deutschen Gegenwartssprache. Tübingen: Niemeyer.

Glaap, Albert-Reiner (1985): „Idiomatisches Englisch = Besseres Englisch?“ Sprache und Literatur in Wissenschaft und Unterricht 2: 95-104. 
Gülich, Elisabeth/Krafft, Ulrich (1992): „“Ich mag es besser”. Konversationelle Bearbeitung vorgeformter Ausdrücke in Gesprächen zwischen deutschen und französischen Sprechern“. Fremdsprachen Lehren und Lernen: 65-87.

Günthner, Susanne (1991): "'A Language with Taste': Uses of Proverbial Sayings in Intercultural Communication". Text 11/3: 399-418.

Häcki Buhofer (2007): "Phraseme im Erstspracherwerb”. In: Burger, Harald/Dobrovol'skij, Dmitrij/Kühn, Peter/Norrick, Neal R. (eds.): Phraseologie: Ein internationales Handbuch der zeitgenössischen Forschung/Phraseology. An International Handbook of Contemporary Research. Berlin, NewYork, Walter de Gruyter: 854-869. (=Handbücher zur Sprach- und Kommunikationswissenschaft/ Handbooks of Linguistics and Communication Science 28.1-28.2).

Häcki Buhofer, Annelies/Burger, Harald (1994): „Phraseologismen im Urteil von Sprecherinnen und Sprechern“. In: Sandig, Barbara (ed.): EUROPHRAS 92 Tendenzen der Phraseologieforschung. Bochum, Brockmeyer: 1-33 (= Studien zur Phraseologie und Paromiologie 1).

Hallsteinsdóttir, Erla (1999): „Indizien der Phraseologizität. Erkennen und Verstehen von fremdsprachlichen Phraseologismen am Beispiel Deutsch als Fremdsprache“.Deutsch als Fremdsprache 2: 93-97.

Hallsteinsdóttir, Erla (2001): Das Verstehen idiomatischer Phraseologismen in der Fremdsprache Deutsch. Hamburg: Verlag Dr. Kovač (= PHILOLOGIA Sprachwissenschaftliche Forschungsergebnisse 49). http://www.verlagdrkovac.de/volltexte/3-8300-0435-4.htm (gesehen am 24. November 2010).

Hallsteinsdóttir, Erla (2006): "Phraseographie". HERMES Journal of Language and Communication Studies 36: 91-128. http://download2.hermes.asb.dk/archive/download/Hermes-36-Hallsteinsdottir.pdf (gesehen am 24. November 2010).

Hallsteinsdóttir, Erla (2011): „Phraseological comptence and the translation of phrasemes”. In: Pamies, Antonio/Luque-Nadal, Lucía/Pazos, José-Manuel (eds.): Multi-lingual Phraseography: Translation and learning applications. Baltmannsweiler: Schneider Verlag.

Hallsteinsdóttir, Erla/Farø, Ken (2006): „Neue theoretische und methodische Ansätze in der Phraseologieforschung“. Linguistik online 27: 3-10.

Hallsteinsdóttir, Erla/Farø, Ken (2010): "Interlinguale Phraseologie: Theorie, Praxis und Perspektiven". Yearbook of Phraseology: 125-158.

Hallsteinsdóttir, Erla/Šajanková, Monika/Quasthoff, Uwe (2006): „Vorschlag eines phraseologischen Optimums für Deutsch als Fremdsprache auf der Basis von Frequenzuntersuchungen und Geläufigkeitsbestimmungen". Linguistik-online 27, 2/06: 119-138. http://www.linguistik-online.de/27 06/hallsteinsdottir et al.pdf (gesehen am 24. November 2010).

Henk, Katrin (2001): „Phraseologismen in literarischen Texten - ein Unterrichtsbeispiel. In: Lorenz-Bourjot, Martine/Lüger, Heinz-Helmut (eds.): Phraseologie und Phraseodidaktik. Wien: Edition Praesens: 179-203. (= Beiträge zur Fremdsprachenvermittlung, Sonderheft 4).

Hessky, Regina (1992): „Aspekte der Verwendung von Phraseologismen im Unterricht Deutsch als Fremdsprache“. Fremdsprachen Lehren und Lernen, 159-168.

Hessky, Regina (1997): „"Feste Wendungen - ein heißes Eisen? Einige phraseodidaktische" Überlegungen für den DaF-Unterricht“". Deutsch als Fremdsprache 3, 139-143.

Hirschfeld, Ursula (1996): „Der Ton macht die Musik. Phonetik und Phraseologie“. Fremdsprache Deutsch 15: 31-35.

Holzinger, Herbert J. (1998): „Kontrastive Phraseologie und Deutsch als Fremdsprache für Spanischsprechende“. In: Eismann, Wolfgang (ed.): Europhras 95. Europäische Phraseo- 
logie im Vergleich: Gemeinsames Erbe und kulturelle Vielfalt. Bochum, Brockmeyer: 337350. (= Studien zur Phraseologie und Parömiologie 15).

Jesenšek, Vida (2006): „Phraseologie und Fremdsprachenlernen. Zur Problematik einer angemessenen phraseodidaktischen Umsetzung“. Linguistik online 27: 137-147. http://www.linguistik-online.de/27_06/jerensek.pdf (gesehen am 28. September 2010).

Jesenšek, Vida/Fabčič, Melanija (2007) (eds.): Phraseologie kontrastiv und didaktisch. Neue Ansätze in der Fremdsprachenvermittlung. Maribor: Slavistično društvo: Filozofska fakulteta (= Zora 47).

Koller, Werner (1987): „Überlegungen zu einem Phraseologie-Wörterbuch für Fremdsprachenunterricht und Übersetzungspraxis“. In: Burger, Harald/Zett, Robert (eds.): Aktuelle Probleme der Phraseologie. Bern, Peter Lang: 109-120.

Köster, Lutz (2001): „Vorsicht: Sie könnten andere mit Ihren Ansprüchen vor den Kopf stoßen. Phraseologismen in populären Kleintexten und ihr Einsatz im DaF-Unterricht“. In: Lorenz-Bourjot, Martine/Lüger, Heinz-Helmut (eds.): Phraseologie und Phraseodidaktik. Wien: Edition Praesens: 137-153. (= Beiträge zur Fremdsprachenvermittlung, Sonderheft 4).

Korhonen, Jarmo/Wotjak, Barbara (2001):.”Kontrastivität in der Phraseologie”. In: Helbig, Gerhard/Gotze, Lutz/Henrici, Gert (eds.): Deutsch als Fremdsprache: ein internationales Handbuch. Berlin, de Gruyter: 224-235.

Kühn, Peter (1992): „Phraseodidaktik. Entwicklungen, Probleme und Überlegungen für den Muttersprachenunterricht und den Unterricht DaF“. Fremdsprachen Lehren und Lernen 21: 169-189.

Kühn, Peter (1994): „Pragmatische Phraseologie: Konsequenzen für die Phraseographie und Phraseodidaktik“. In: Sandig, Barbara (ed.): EUROPHRAS 92 Tendenzen der Phraseologieforschung. Bochum, Brockmeyer: 411-428 (= Studien zur Phraseologie und Paromiologie 1).

Kühn, Peter (2007): „Phraseme im Muttersprachenunterricht“. In: Burger, Harald/Dobrovol'skij, Dmitrij/Kühn, Peter/Norrick, Neal R. (eds.): Phraseologie: Ein internationales Handbuch der zeitgenössischen Forschung/Phraseology. An International Handbook of Contemporary Research. Berlin, NewYork, Walter de Gruyter: 881-893. (=Handbücher zur Sprach- und Kommunikationswissenschaft/ Handbooks of Linguistics and Communication Science 28.1-28.2).

Kühnert, Helmut (1985): „Die Rolle des Bildverständnisses bei Phraseologismen im Fremdsprachenunterricht für Fortgeschrittene“. Deutsch als Fremdsprache 22: 223-227.

Laskowski, Marek (2010) „Phraseodidaktische Einsatzmöglichkeiten von Fernsehwerbung im DaF-Unterricht"“. In: Korhonen, Jarmo/Mieder, Wolfgang/Piirainen, Elisabeth/Piñel, Rosa (eds.): EUROPHRAS 2008 Beiträge zur internationalen Phraseologiekonferenz vom 13.16.8.2008 in Helsinki. Helsinki, Universität Helsinki: 399-410. http://www.helsinki.fi/deutsch/europhras/ep2008.pdf (gesehen am 24. September 2010).

Lenk, Hartmut E. H. (2010): „Phraseologismen im Austro-, Deutsch- und Ostrock. Exemplarische Analysen zur deutschsprachigen Rocklyrik der 80er Jahre“. In: Korhonen, Jarmo/Mieder, Wolfgang/Piirainen, Elisabeth/Piñel, Rosa (eds.): EUROPHRAS 2008 Beiträge zur internationalen Phraseologiekonferenz vom 13.-16.8.2008 in Helsinki. Helsinki, Universität Helsinki: 226-236. http://www.helsinki.fi/deutsch/europhras/ep2008.pdf (gesehen am 24. September 2010).

Lenk, Hartmut E.H. (2001): „Phraseologie des Deutschrock als Gegenstand des DaFUnterrichts“. In: Lorenz-Bourjot, Martine/Lüger, Heinz-Helmut (eds.): Phraseologie und Phraseodidaktik. Wien: Edition Praesens: 155-178. $\quad(=$ Beiträge zur Fremdsprachenvermittlung, Sonderheft 4). 
Lipold, Günter (1998): „... wie der Hase läuft. Gibt es Dekodierungssignale bei Phraseolexemen?" In: Ernst, Peter/Patocka, Franz (eds.): Deutsche Sprache in Raum und Zeit: Festschrift für Peter Wiesinger zum 60. Geburtstag. Wien: Edition Praesens, 297-310.

Lorenz-Bourjot, Martine/Lüger, Heinz-Helmut (2001) (eds.): Phraseologie und Phraseodidaktik. Wien: Edition Praesens. (= Beiträge zur Fremdsprachenvermittlung, Sonderheft 4).

Lüger, Heinz-Helmut (1997): „Anregungen zur Phraseodidaktik“. Beiträge zur Fremdsprachenvermittlung $\quad 32: \quad 69-120 . \quad \mathrm{http}: / / \mathrm{www} . u b . u n i-$ konstanz.de/kops/volltexte/1999/295/pdf/295_1.pdf (gesehen am 24. November 2010).

Lüger, Heinz-Helmut (1997a): „Auswahlbibliographie zur Phraseodidaktik“. Beiträge zur Fremdsprachenvermittlung 32: 121-127. $\quad 3 t$ h://www.ub.unikonstanz.de/kops/volltexte/1999/295/pdf/295_1.pdf (gesehen am 24. November 2010).

Posner, Roland/Robering, Klaus/Sebeok, Thomas A. (1997-2003) (eds.): Semiotik: ein Handbuch zu den zeichentheoretischen Grndlagen von Natur und Kultur/Semiotics. A handbook on the sign-theoretic foundations of nature and culture. Vol. 1-3. Berlin: de Gruyter. (=Handbücher zur Sprach- und Kommunikationswissenschaft 13:1-3).

Ptashnyk, Stefaniya (2009): Phraseologische Modifikationen und ihre Funktionen im Text. Eine Studie am Beispiel der deutschsprachigen Presse. Baltmannsweiler: Schneider Verlag Hohengehren.

Quasthoff, Uwe/Schmidt, Fabian/Hallsteinsdóttir, Erla (2010): „Häufigkeit und Struktur von Phraseologismen am Beispiel verschiedener Web-Korpora“. In: Ptashnyk, Stefaniya/Hallsteinsdóttir, Erla/Bubenhofer, Noah (eds.): Korpora, Web und Datenbanken. Computergestützte Methoden in der modernen Phraseologie und Lexikographie. Baltmannsweiler, Schneider Verlag Hohengehren: 37-53. (= Phraseologie und Parämiologie 25).

Rabanus, Stefan/Smolka, Eva/Streb, Judith/Rösler, Frank (2008): "Die mentale Verarbeitung von Verben in idiomatischen Konstruktionen". Zeitschrift für Germanistische Linguistik 36: 27-47.

Reder, Anna (2006): Kollokationen in der Wortschatzarbeit. Wien: Praesens.

Reder, Anna (2008): "Erkennen DaF-Lernende Kollokationen?" In: Scheibl, György (ed.): Tests im DaF-Unterricht - DaF-Unterricht im Test. Szeged, Grimm: 105-115.

Rentel, Nadine (2005): Bild und Sprache in der Werbung. Die Formale und inhaltliche Konnexion von verbalem und visuellem Teiltext in der französischen Anzeigenwerbung der Gegenwart. Frankfurt a.M. etc.: Peter Lang.

Roos, Eckhard (1992): „Lehrmaterialien zur englischen Idiomatik - Eine kritische Analyse“. Fremdsprachen Lehren und Lernen 21, 190-201.

Šajanková, Monika (2005): Gebrauch, Erwerb und Verständnis von Phraseologismen. Bratislava: Dissertation an der Philosophischen Fakultät der Comenius Universität Bratislava.

Šajanková, Monika (2005a): "Auswahl der Phraseologismen zur Entwicklung der aktiven phraseologischen Kompetenz". In: Jankoviãová, Milada/Mlacek, Jozef/Skladaná, Jana (eds.): Frazeologicke Študie IV. Bratislava, Veda, Vydatel'stvo Slovenskej Akademie Vied: 325-340.

Sandig, Barbara (2010): „Formulierungsmuster, idiomatische Prägungen, Konstruktionen. Zum Bewerten in journalistischen Texten“. In: Korhonen, Jarmo/Mieder, Wolfgang/Piirainen, Elisabeth/Piñel, Rosa (eds.): EUROPHRAS 2008 Beiträge zur internationalen Phraseologiekonferenz vom 13.-16.8.2008 in Helsinki. Helsinki, Universität Helsinki: 169-179. http://www.helsinki.fi/deutsch/europhras/ep2008.pdf (gesehen am 24. September 2010).

Schmale, Günter (2009): „Phraseologische Ausdrücke als Bestandteil des Fremdsprachenerwerbs. Überlegungen zur Phraseodidaktik auf der Grundlage einer 
korpusbasierten Analyse deutscher Talkshows“. Beiträge zur Fremdsprachenvermittlung 48: 149-179.

Stedje, Astrid (1987): „Sprecherstrategien im Spiegel der Phraseologie“. In: Korhonen, Jarmo (ed.): Beiträge zur allgemeinen und germanistischen Phraseologieforschung. Oulu, Universität Oulu: 91-110. (= Veröffentlichungen des Germanistischen Instituts, 7).

Steyer, Kathrin (2003): "Korpus, Statistik, Kookkurrenz. Lässt sich Idiomatisches "berechnen"?" In: Burger, Harald/Häcki Buhofer, Annelies/Gréciano, Gertrud (eds.): Flut von Texten - Vielfalt der Kulturen. Baltmannsweiler, Schneider Verlag Hohengehren: 3346. (= Phraseologie und Parömiologie 14).

Stolze, Peter (2001): „Geflügelte Worte. Möglichkeiten der Unterrichtlichen Integration“. In: Lorenz-Bourjot, Martine/Lüger, Heinz-Helmut (eds.): Phraseologie und Phraseodidaktik. Wien: Edition Praesens: 125-135. (= Beiträge zur Fremdsprachenvermittlung, Sonderheft 4).

Ulbricht, Adelheid (1989): „Idiomatische Wendungen im Fremdsprachenunterricht Deutsch“. Deutsch als Fremdsprache 26: 98-103.

Vajičková, Mária (2010): „Textgestaltende Funktionen von Phrasemen in der Presse“. In: Korhonen, Jarmo/Mieder, Wolfgang/Piirainen, Elisabeth/Piñel, Rosa (eds.): EUROPHRAS 2008 Beiträge zur internationalen Phraseologiekonferenz vom 13.-16.8.2008 in Helsinki. Helsinki, Universität Helsinki: 180-190. http://www.helsinki.fi/deutsch/europhras/ep2008.pdf (gesehen am 24. September 2010).

Weller, Franz-Rudolf (1979): “"Idiomatizität” als didaktisches Problem des Fremdsprachenunterrichts - erläutert am Beispiel des Französischen“. Die Neueren Sprachen 78/6: 530554.

Weller, Franz-Rudolf (1992): „Wie 'authentisch' ist 'idiomatisches' Französisch? Anmerkungen zu zwei unklaren Begriffen der Fremdsprachendidaktik“. Fremdsprachen Lehren und Lernen 21: 117-139.

Wotjak, Barbara (1992): Verbale Phraseolexeme in System und Text. Tübingen: Niemeyer. Wotjak, Barbara (1996) (ed.): Fremdsprache Deutsch 15: Redewendungen und Sprichwörter. Wray, Allison (2007): "Set phrases in second language acquisition". In: Burger, Harald/Dobrovol'skij, Dmitrij/Kühn, Peter/Norrick, Neal R. (eds.): Phraseologie: Ein internationales Handbuch der zeitgenössischen Forschung/Phraseology. An International Handbook of Contemporary Research. Berlin, NewYork, Walter de Gruyter: 870-881. (=Handbücher zur Sprach- und Kommunikationswissenschaft/ Handbooks of Linguistics and Communication Science 28.1-28.2). 